\title{
Glucocorticoid signaling in pancreatic islets modulates gene regulatory programs and genetic risk of type 2 diabetes
}

\author{
Anthony Aylward $\oplus^{1 \oplus}$, Mei-Lin Okino ${ }^{2 \odot}$, Paola Benaglio ${ }^{2}$, Joshua Chiou $\oplus^{3}$, Elisha Beebe ${ }^{2}$, \\ Jose Andres Padilla $\oplus^{2}$, Sharlene Diep ${ }^{2}$, Kyle J. Gaulton $\circledast^{2,4 *}$ \\ 1 Bioinformatics and Systems Biology graduate program, University of California San Diego, La Jolla, \\ California, United States of America, 2 Department of Pediatrics, University of California San Diego, La Jolla, \\ California, United States of America, 3 Biomedical Sciences graduate program, University of California San \\ Diego, La Jolla, California, United States of America, 4 Institute for Genomic Medicine, University of \\ California San Diego, La Jolla, California, United States of America \\ - These authors contributed equally to this work. \\ *kgaulton@ucsd.edu
}

\section{Open ACCess}

Citation: Aylward A, Okino M-L, Benaglio P, Chiou J, Beebe E, Padilla JA, et al. (2021) Glucocorticoid signaling in pancreatic islets modulates gene regulatory programs and genetic risk of type 2 diabetes. PLoS Genet 17(5): e1009531. https://doi. org/10.1371/journal.pgen.1009531

Editor: Michael L. Stitzel, Jackson Laboratory, UNITED STATES

Received: May 22, 2020

Accepted: April 6, 2021

Published: May 13, 2021

Peer Review History: PLOS recognizes the benefits of transparency in the peer review process; therefore, we enable the publication of all of the content of peer review and author responses alongside final, published articles. The editorial history of this article is available here: https://doi.org/10.1371/journal.pgen.1009531

Copyright: ๑ 2021 Aylward et al. This is an open access article distributed under the terms of the Creative Commons Attribution License, which permits unrestricted use, distribution, and reproduction in any medium, provided the original author and source are credited.

Data Availability Statement: The authors confirm that all data underlying the findings are fully available without restriction. All raw data are available from the GEO database (GSE167250). All

\section{Abstract}

Glucocorticoids are key regulators of glucose homeostasis and pancreatic islet function, but the gene regulatory programs driving responses to glucocorticoid signaling in islets and the contribution of these programs to diabetes risk are unknown. In this study we used ATACseq and RNA-seq to map chromatin accessibility and gene expression from eleven primary human islet samples cultured in vitro with the glucocorticoid dexamethasone at multiple doses and durations. We identified thousands of accessible chromatin sites and genes with significant changes in activity in response to glucocorticoids. Chromatin sites up-regulated in glucocorticoid signaling were prominently enriched for glucocorticoid receptor binding sites and up-regulated genes were enriched for ion transport and lipid metabolism, whereas down-regulated chromatin sites and genes were enriched for inflammatory, stress response and proliferative processes. Genetic variants associated with glucose levels and T2D risk were enriched in glucocorticoid-responsive chromatin sites, including fine-mapped variants at 51 known signals. Among fine-mapped variants in glucocorticoid-responsive chromatin, a likely casual variant at the $2 \mathrm{p} 21$ locus had glucocorticoid-dependent allelic effects on beta cell enhancer activity and affected $S I X 2$ and $S I X 3$ expression. Our results provide a comprehensive map of islet regulatory programs in response to glucocorticoids through which we uncover a role for islet glucocorticoid signaling in mediating genetic risk of T2D.

\section{Author summary}

Glucocorticoids regulate inflammation and metabolism and are widely used in the treatment of immune disorders, although prolonged exposure to glucocorticoids can lead to the development of diabetes. In this study we determined the response of primary pancreatic islets, which are central to the development of diabetes, to the glucocorticoid dexamethasone at multiple doses and durations. We observed widespread changes in 
data underlying graphs are provided in the main text or as Supporting Information.

Funding: This work was supported by National Institute of Diabetes and Digestive and Kidney Diseases awards DK114650, DK122607, and DK120429 to KJG. This publication includes data generated at the UC San Diego IGM Genomics Center utilizing an Illumina NovaSeq 6000 that was purchased with funding from a National Institutes of Health SIG grant (\#S10 0D026929). The funders had no role in study design, data collection and analysis, decision to publish, or preparation of the manuscript.

Competing interests: I have read the journal's policy and the authors of this manuscript have the following competing interests: $\mathrm{KJG}$ is a consultant for Genentech and holds stock in Vertex Pharmaceuticals pancreatic islets after glucocorticoid treatment at glucocorticoid receptor binding sites, as well as at key genes involved in islet function and processes related to steroid and lipid metabolism, ion channel activity, inflammation, and growth. Genetic variants affecting type 2 diabetes and glucose levels were located in sites affected by glucocorticoids at many genomic regions, and highlighted genes regulated by these sites through which glucocorticoid signaling may contribute directly to the development of diabetes. Together these results provide key insight into how glucocorticoid treatment affects pancreatic islet function and risk of diabetes.

\section{Introduction}

Glucocorticoids are steroid hormones produced by the adrenal cortex which broadly regulate inflammatory, metabolic and stress responses and are widely used in the treatment of immune disorders [1-3]. The metabolic consequences of glucocorticoid action are directly relevant to diabetes pathogenesis, as chronic glucocorticoid exposure causes hyperglycemia and steroidinduced diabetes and endogenous excess of glucocorticoids causes Cushing's syndrome in which diabetes is a common co-morbidity [4,5]. Glucocorticoids contribute to the development of diabetes both through insulin resistance and obesity via effects on adipose, liver and muscle, as well as through pancreatic islet dysfunction [4]. In islets, glucocorticoid signaling has been shown to modulate numerous processes such as insulin secretion, ion channel activity, cAMP signaling, proliferation and development [6-11].

The effects of glucocorticoids on cellular function are largely mediated through regulation of transcriptional activity. Glucocorticoids diffuse through the cell membrane into cytoplasm and bind the glucocorticoid receptor (GR), which is then translocated into the nucleus where it binds DNA and modulates the transcriptional program [12-15]. Gene activity can be affected by GR via direct genomic binding and regulation as well as indirectly through physical interaction with other transcriptional regulators [13-17]. Previous studies have profiled glucocorticoid signaling by mapping genomic locations of GR binding and other epigenomic features such as histone modifications and chromatin accessibility in response to endogenous glucocorticoids such as cortisol or analogs such as dexamethasone $[13,14,18,19]$. Studies have also shown that the genomic function of GR is largely mediated via binding to regions of accessible chromatin [20,21].

Genetic studies have identified hundreds of genomic loci that contribute to diabetes risk and which primarily map to non-coding sequence and affect gene regulation [22-25]. Risk variants for type 2 diabetes (T2D) are enriched for pancreatic islet regulatory sites [2224,26,27], while type 1 diabetes (T1D) risk variants are enriched for immune cell as well as islet regulatory sites. The specific mechanisms of most risk variants in islets are unknown, however, which is critical for understanding the genes and pathways involved in disease pathogenesis and for the development of novel therapeutic strategies. Previous studies of islet chromatin have focused predominantly on normal, non-disease states [27-33], although recent evidence has shown that diabetes risk variants can interact with environmental stimuli to affect islet chromatin and gene regulatory programs [34].

The effects of glucocorticoid and other steroid hormone signaling on islet regulatory programs and how these signals interact with diabetes risk variants, however, are largely unknown. In this study we profiled islet accessible chromatin and gene expression in primary human pancreatic islets exposed in vitro to the glucocorticoid dexamethasone. Glucocorticoid signaling had widespread effects on islet accessible chromatin and gene expression levels. Up- 
regulated chromatin sites were strongly enriched for glucocorticoid receptor binding and upregulated genes were enriched for processes related to ion channel activity and steroid and lipid metabolism. Conversely, down-regulated sites and genes were involved in inflammation, stress response and proliferation. Genetic variants affecting T2D risk and glucose levels were significantly enriched in glucocorticoid-responsive chromatin sites, including a likely causal variant at the SIX2/3 locus which had glucocorticoid-dependent effects on beta cell enhancer activity and affected $S I X 2$ and SIX 3 expression. Together our results provide a comprehensive map of islet gene regulatory programs in response to glucocorticoids which will facilitate a greater mechanistic understanding of glucocorticoid signaling and its role in islet function and diabetes risk.

\section{Results}

\section{Map of gene regulation in pancreatic islets in response to glucocorticoid signaling}

In order to determine the effects of glucocorticoid signaling on pancreatic islet regulation, we cultured primary islet cells in vitro with dexamethasone at several different doses $(100 \mathrm{ng} / \mathrm{mL}$ for $24 \mathrm{hr}, 4 \mathrm{ng} / \mathrm{mL}$ for $6 \mathrm{hr}$ and $24 \mathrm{hr}$ ) as well as in untreated conditions and measured accessible chromatin and gene expression levels in both treated and untreated cells. An overview of the study design is provided in Fig 1A.

We assayed gene expression in dexamethasone-treated and untreated islets from 6 total samples using RNA-seq (S1 Table; see Methods). Across replicate samples we observed changes in expression levels of genes both known to be induced by dexamethasone such as ZBTB16 [35-37] and VIPR1 [38] as well as those suppressed by dexamethasone such as IL11 [39] in both the high-dose $(100 \mathrm{ng} / \mathrm{mL})$ and low-dose $(4 \mathrm{ng} / \mathrm{mL})$ treatments (Figs 1B, 1C, S1A, $\mathrm{S} 1 \mathrm{~B}$ and $\mathrm{S1C}$ ). We next assayed accessible chromatin in dexamethasone-treated and untreated islets from 9 total samples using ATAC-seq (S1 Table; see Methods). Across replicate samples we observed reproducible changes in islet accessible chromatin signal concordant with changes in gene expression. For example, accessible chromatin signal was notably induced at several sites proximal to the ZBTB16 and VIPR1 genes in dexamethasone-treated compared to untreated islets in both high- and low-dose treatments (Figs 1D, 1E, S2, S3 and S4). Similarly, accessible chromatin signal was reduced at a site proximal to the $I L 11$ promoter in glucocorticoid-treated compared to untreated islets (S5 Fig).

\section{Islet accessible chromatin sites with differential activity in response to glucocorticoid signaling}

To understand the effects of glucocorticoid signaling on accessible chromatin in islets at a genome-wide level, we first performed principal components analysis (PCA) using normalized read counts in chromatin sites for each treated and untreated islet ATAC-seq sample (see Methods). We observed reproducible differences in accessible chromatin profiles in dexamethasone-treated compared to untreated islets across replicate samples, where the effects of lowdose treatment $(4 \mathrm{ng} / \mathrm{mL}, \mathrm{n}=3)$ were intermediate to high-dose treatment $(100 \mathrm{ng} / \mathrm{mL}, \mathrm{n}=6)$ relative to untreated samples $(\mathrm{n}=9)($ Fig $2 \mathrm{~A})$.

We then identified specific islet accessible chromatin sites with significant differential activity in glucocorticoid treatment compared to untreated control cells. We first defined a canonical set of 127,228 islet accessible chromatin sites genome-wide by comparing replicate samples using IDR (see Methods, S2 Table). Among these canonical sites, there were 2,688 sites with significant evidence $($ FDR $<.10)$ for differential activity in glucocorticoid signaling at high- 
A

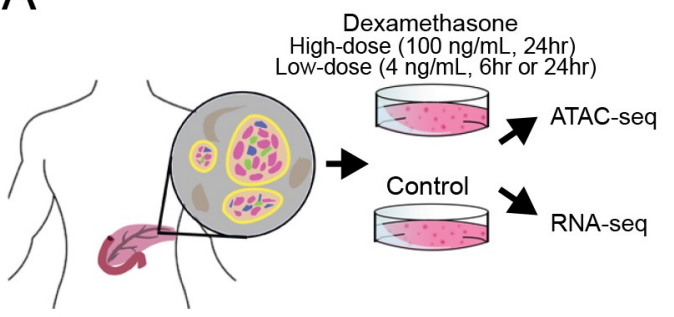

D
B

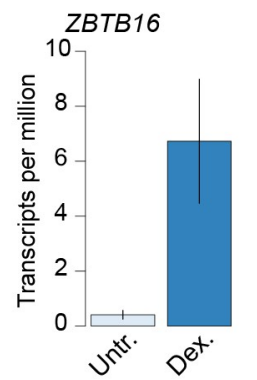

C

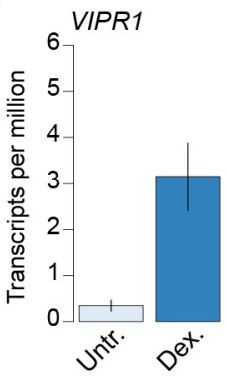

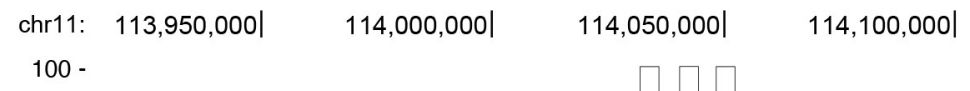

Dex.
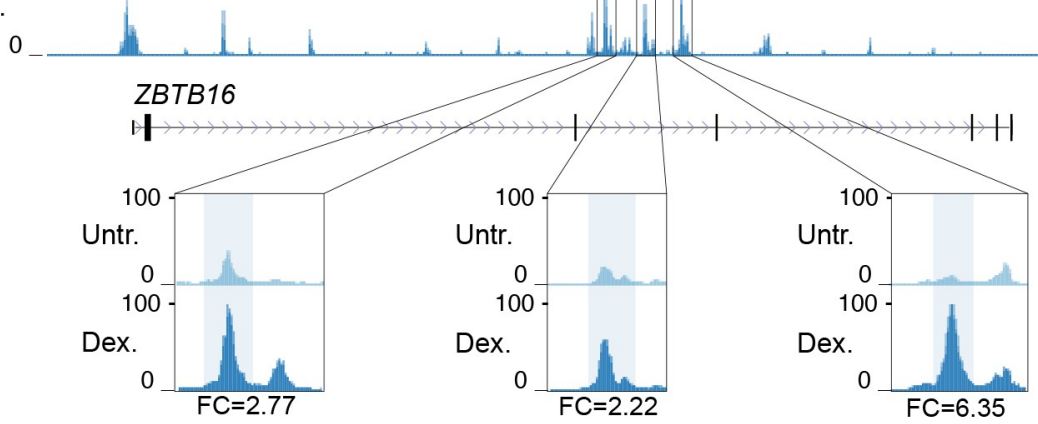

$E$

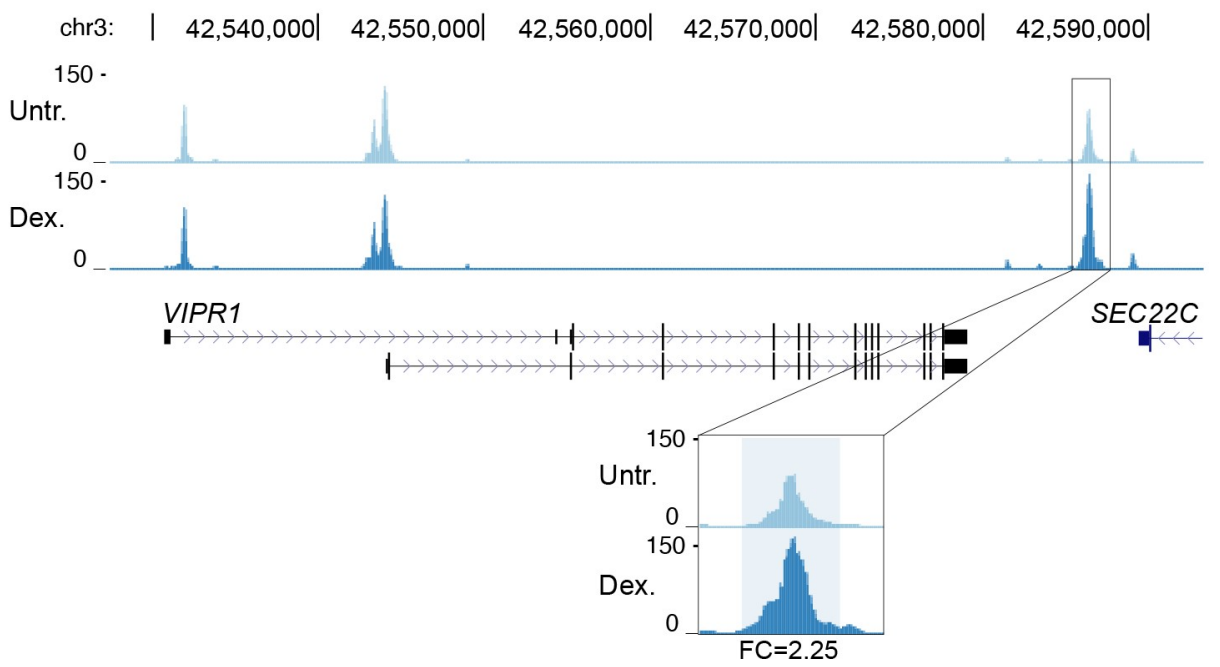

Fig 1. A map of gene regulation in pancreatic islets in response to glucocorticoid signaling. (A) Overview of study design. Primary pancreatic islet samples were split and separately cultured in normal conditions and including the glucocorticoid dexamethasone at either a high-dose (100ng/mL for $24 \mathrm{hr}$ ) or low-dose ( $4 \mathrm{ng} / \mathrm{mL}$ for $6 \mathrm{hr}$ or $24 \mathrm{hr}$ ) treatment, and then profiled for gene expression and accessible chromatin using RNA-seq and ATAC-seq assays. Genes with known induction in glucocorticoid signaling (B) ZBTB16 and (C) VIPR1 had increased expression in glucocorticoid-treated islets compared to untreated islets. Values represent mean and standard error. (D) At the ZBTB16 locus several accessible chromatin sites intronic to ZBTB16 had increased accessibility in glucocorticoid treated (Dex.) compared to untreated (Untr.) islets. (E) At the VIPR1 locus an accessible chromatin site downstream of VIPR1 had increased accessibility in glucocorticoid treated (Dex.) compared to untreated (Untr.) islets. Values in D and E represent RPKM normalized ATAC-seq read counts. Fold-change (FC) in accessible chromatin signal in glucocorticoid treatment compared to untreated indicated at highlighted sites. All results shown are for the high-dose treatment. 
A
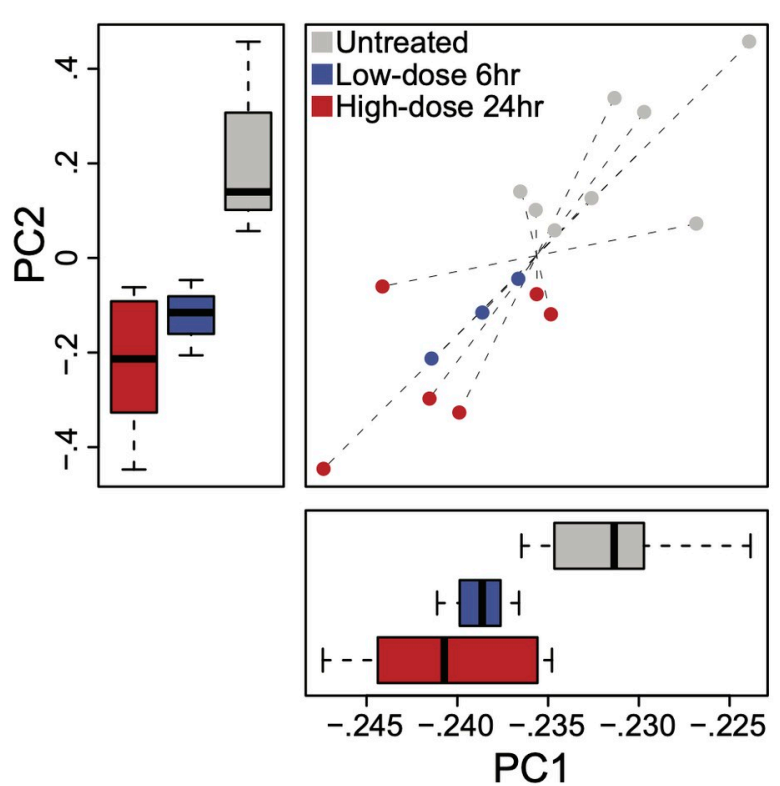

B

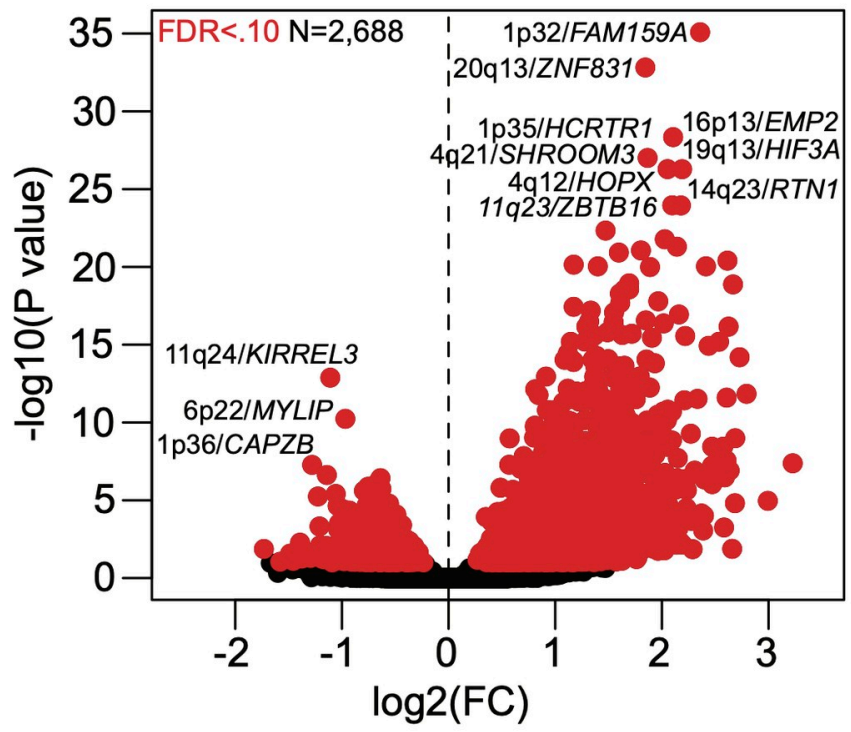

C

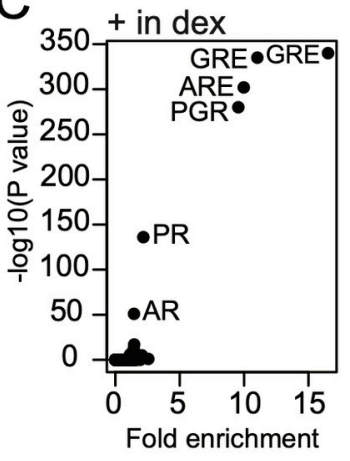

$E$

chr2 $45,160,000|45,170,000| 45,180,000|45,190,000|$

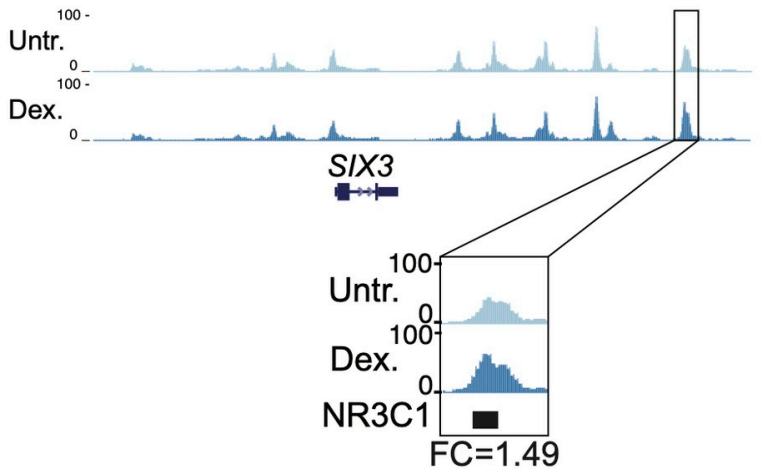

D

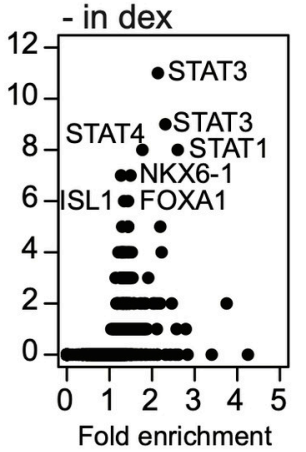

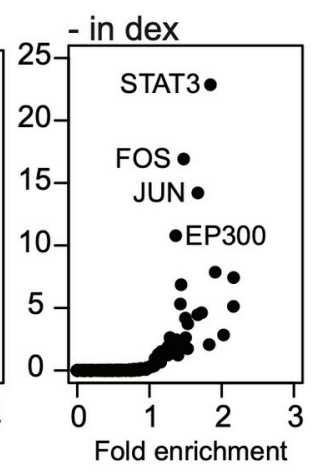

F

G
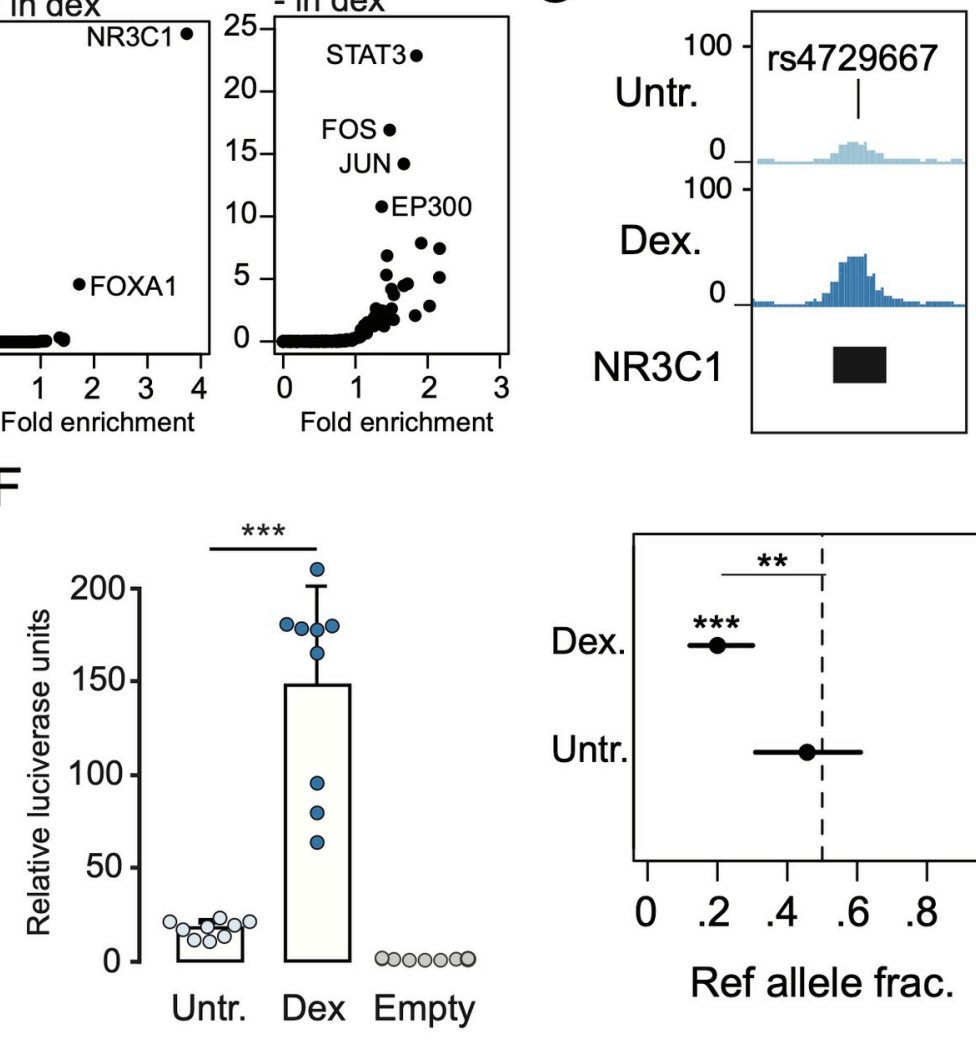

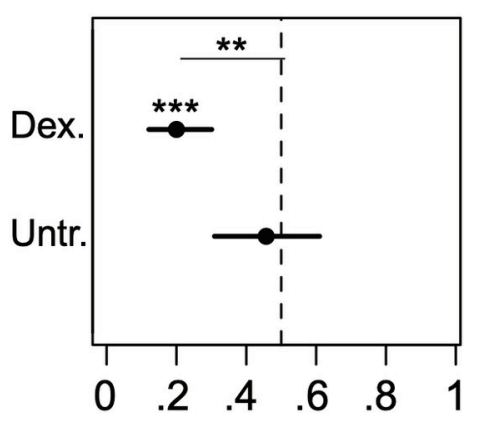

Ref allele frac.

Fig 2. Glucocorticoid signaling affects chromatin accessibility in pancreatic islets. (A) Principal components plot showing ATAC-seq signal for high-dose (red) and low-dose (blue) glucocorticoid-treated islets and untreated (grey) islets from 9 total donors. Dashed lines connect assays from the same sample, and box plots on each axis represent the distribution of principal components of samples for each condition. (B) Volcano plot of sites with differential chromatin accessibility in glucocorticoid treated compared to untreated islets. Sites with significant differential activity (FDR $<.10)$ are highlighted in red. The sites with the most significant changes are labelled with the locus and the nearest gene. (C) Transcription factor (TF) sequence motifs enriched in differential chromatin sites with increased activity ( + in dex) and decreased activity (- in dex) in glucocorticoid-treated islets. (D) Enrichment of ChIP-seq sites from ENCODE for 160 TFs in differential chromatin sites with increased activity ( + in dex) and decreased activity (- in dex) in glucocorticoid-treated islets. (E) A chromatin site at the SIX2/3 locus had increased activity in glucocorticoid-treated islets and overlapped a ChIP-seq site for the glucocorticoid receptor (GR/NR3C1) (top). Fold-change (FC) in accessible chromatin signal in glucocorticoid treatment compared to untreated indicated at the highlighted site for high-dose treatment. (F) The differential site at SIX2/3 had glucocorticoid- 
dependent effects on enhancer activity in gene reporter assays in MIN6 cells (bottom). Values represent mean and standard deviation. (G) Variant rs4729667 mapped in a chromatin site with increased activity in glucocorticoid-treated islets and had stronger allelic imbalance in chromatin accessibility in glucocorticoidtreated compared to untreated islets. Values represent ref allele fraction and $95 \%$ confidence intervals. For panels B, C and D the values shown are from results using high-dose treatment. ${ }^{* *} \mathrm{P}<.01,{ }^{* * *} \mathrm{P}<1 \times 10^{-4}$.

https://doi.org/10.1371/journal.pgen.1009531.g002

dose treatment (Fig 2B and S3 Table). Among these 2,688 glucocorticoid-responsive sites, 1,992 had up-regulated activity and 695 had down-regulated activity in glucocorticoid treated compared to untreated cells (Fig 2B and S3 Table). The majority of sites (95\%) with differential activity were already accessible in untreated islets, suggesting that sites induced by glucocorticoid signaling are typically not activated de novo. Furthermore, a majority of differentially accessible sites $(2,453,91 \%)$ were not proximal to promoter regions, suggesting they act via distal regulation of gene activity. At low-dose treatment, 373 sites had differential activity (FDR $<.10)$ in glucocorticoid signaling, where the majority (350) were up-regulated (S3 Table). Among sites with differential activity in either treatment, the effects in high- and lowdose were highly concordant (Spearman $\rho=.72, \mathrm{P}<2.2 \times 10^{-16}$ ) (S6A and S6B Fig).

We next characterized transcriptional regulators underlying changes in glucocorticoidresponsive islet chromatin. First, we identified TF motifs enriched in genomic sequence underneath sites up-regulated and down-regulated in glucocorticoid-treated islets (see Methods). The most enriched sequence motifs in up-regulated sites for both high- and low-dose treatment were for glucocorticoid and other steroid hormone response elements (high-dose: GRE $\mathrm{P}=1 \times 10^{-340}$, ARE $\mathrm{P}=1 \times 10^{-302}$, PGR $\mathrm{P}=1 \times 10^{-280}$; low-dose: GRE $\mathrm{P}=1 \times 10^{-73}$, ARE $\left.\mathrm{P}=1 \times 10^{-66}, \mathrm{PGR} \mathrm{P}=1 \times 10^{-62}\right)$, in addition to lesser enrichment for TFs relevant to islet function (FOXA1: high-dose $\mathrm{P}=1 \times 10^{-5}$, low-dose $\mathrm{P}=1 \times 10^{-3}$ ) (Fig 2C and S4 Table). Conversely, down-regulated sites in high-dose treatment were most enriched for sequence motifs for STAT TFs (STAT3 $\mathrm{P}=1 \times 10^{-9}$, STAT1 $\mathrm{P}=1 \times 10^{-8}$ ) followed by TFs involved in islet function (NKX6.1 $\mathrm{P}=1 \times 10^{-7}$, FOXA1 $\mathrm{P}=1 \times 10^{-6}$ ) (Fig $2 \mathrm{C}$ and $\mathrm{S} 4$ Table). Next, we determined enrichment of glucocorticoid-responsive chromatin sites for ChIP-seq TF-binding sites previously identified by the ENCODE project. We observed strongest enrichment of up-regulated accessible chromatin sites in both high- and low-dose treatment for glucocorticoid receptor (NR3C1) binding sites (high-dose ratio $=3.7, \mathrm{P}=1.7 \times 10^{-294}$, low-dose ratio $=5.4, \mathrm{P}=2.1 \times 10^{-}$ ${ }^{129}$ ), and less pronounced enrichment for binding sites of FOXA1 (high-dose ratio $=1.7$, $\mathrm{P}=1.6 \times 10^{-55}$; low-dose ratio $=2.3, \mathrm{P}=2.3 \times 10^{-30}$ ) and other TFs (Fig 2D and S4 Table). Downregulated sites were most enriched for STAT binding (STAT3 ratio $=2.1, \mathrm{P}=7.6 \times 10^{-41}$ ) as well as enhancer binding TFs such as FOS/JUN (FOS ratio $=1.5, \mathrm{P}=2.3 \times 10^{-17}$; JUN ratio $=1.7$, $\mathrm{P}=1.3 \times 10^{-14}$ ) and $\mathrm{P} 300\left(\right.$ ratio $\left.=1.4, \mathrm{P}=2.9 \times 10^{-11}\right)$ (Fig 2D and S4 Table).

Accessible chromatin sites with significant up-regulation in glucocorticoid signaling compared to untreated islets included a site that mapped to the SIX2/SIX3 locus (Fig 2E and S3 Table), which also harbors genetic variants associated with fasting glucose level and risk of T2D. The glucocorticoid-responsive site at this locus also directly overlapped a NR3C1 ChIPseq site identified by the ENCODE project (Fig 2E). We tested the glucocorticoid-induced site at this locus (high-dose fold-change $=1.49 ; \mathrm{P}=1.0 \times 10^{-5} ;$ low-dose fold-change $=1.51$; $\mathrm{P}=4.4 \times 10^{-4}$ ) for enhancer activity in luciferase gene reporter assays in dexamethasone-treated and untreated MIN6 mouse insulinoma cells. We observed a significant increase in enhancer activity in dexamethasone-treated cells relative to untreated cells (T-test $\mathrm{P}=1.65 \times 10^{-6}$ ) (Fig $2 \mathrm{~F}$ ), confirming that this site is highly induced in response to glucocorticoid signaling.

Environmental stimuli can interact with genetic variation to affect chromatin accessibility and gene regulation. We therefore determined the effects of genetic variants on islet accessible chromatin in both glucocorticoid-treated and untreated conditions using allelic imbalance 
mapping. We performed microarray genotyping of seven islet samples and imputed genotypes into $39 \mathrm{M}$ variants (see Methods). For variants overlapping islet chromatin sites we obtained read counts in samples heterozygote for that variant, corrected for mapping bias using WASP and modeled the resulting counts for imbalance using a beta-binomial test. We then identified variants with evidence $(\mathrm{FDR}<.10)$ for allelic imbalance in accessible chromatin from either glucocorticoid-treated or untreated islets (S5 Table). Among imbalanced variants, we further identified those with significant differences in allelic effects (FDR $<.10$ ) between glucocorticoid-treated and untreated islets (S5 Table, see Methods). For example, variant rs4729667 at 7q22 mapped in a glucocorticoid-responsive site bound by GR and had significantly stronger imbalance in glucocorticoid-treated islets ( $\mathrm{GC}$ ref frac. $=.20$, untr. ref frac. $=.46 ; \mathrm{P}=3.9 \times 10^{-3}$ ) (Fig $2 \mathrm{G}$ and S5 Table). Conversely, variant rs2291583 at 10p12 in a glucocorticoid-responsive site had significantly stronger imbalance in untreated islets $(\mathrm{GC}$ ref frac $=.39$, untr. ref frac. $=$ $\left..28 ; \mathrm{P}=9.6 \times 10^{-4}\right)(\mathrm{S} 5$ Table).

These results demonstrate that glucocorticoid signaling broadly affects accessible chromatin in islets including sites both up-regulated through glucocorticoid receptor activity and downregulated through the activity of STAT and other TFs.

\section{Genes and pathways with differential regulation in islets in response to glucocorticoid signaling}

We next sought to determine the effects of glucocorticoid treatment on gene expression levels. We first performed PCA using gene transcript counts from untreated and dexamethasonetreated islet samples at each treatment dose and duration obtained from RNA-seq assays (see Methods). There were again reproducible differences in expression levels across replicate samples, where the effects of low-dose treatment $(4 \mathrm{ng} / \mathrm{mL}$ at $24 \mathrm{hr}, \mathrm{n}=3 ; 4 \mathrm{ng} / \mathrm{mL}$ at $6 \mathrm{hr}, \mathrm{n}=3$ ) were intermediate to high-dose treatment $(100 \mathrm{ng} / \mathrm{mL}$ at $24 \mathrm{hr}, \mathrm{n}=6)$ relative to untreated samples $(\mathrm{n}=6)($ Fig 3A).

We identified specific genes with differential expression in response to glucocorticoids compared to untreated islet samples using DESeq2 (see Methods). There were 2,837 genes with significant evidence for differential expression $(\mathrm{FDR}<0.10)$ in glucocorticoid signaling at high-dose treatment (S6 Table). Among these genes, 1,348 (47\%) were up-regulated and 1,489 (53\%) were down-regulated in response to glucocorticoids compared to untreated islets (Fig $3 \mathrm{~B})$. Genes with the most significant up-regulation included $E D N 3(\log 2(\mathrm{FC})=1.44, \mathrm{FDR}=$ $\left.2.42 \times 10^{-81}\right)$, FAM115C $\left(\log 2(\mathrm{FC})=1.52, \mathrm{FDR}=3.61 \times 10{ }^{-{ }^{75}}\right)$, METTL7A $(\log 2(\mathrm{FC})=1.81$, $\left.\mathrm{FDR}=4.36 \times 10^{-71}\right), P R R 15 L\left(\log 2(\mathrm{FC})=2.20, \mathrm{FDR}=9.55 \times 10^{-62}\right)$, and CCND3 $(\log 2(\mathrm{FC})=$ $\left.0.95, \mathrm{FDR}=9.05 \times 10^{-60}\right)$. Conversely, genes with most significant down-regulation included PCSK1 $\left(\log 2(\mathrm{FC})=-1.21, \mathrm{FDR}=2.05 \times 10^{-61}\right)$, KLHL41 $\left(\log 2(\mathrm{FC})=-1.31, \mathrm{FDR}=8.84 \times 10^{-59}\right)$, DHRS2 $\left(\log 2(\mathrm{FC})=-1.41, \mathrm{FDR}=2.19 \times 10^{-49}\right)$ and $C D 36\left(\log 2(\mathrm{FC})=-1.21, \mathrm{FDR}=2.41 \times 10^{-49}\right)$ (Fig 3B). At low-dose treatment 775 and 848 genes had differential expression $(F D R<.10)$ at $6 \mathrm{hr}$ and 24hr, respectively (S6 Table and S7A and S7B Fig). Among genes differentially expressed in either treatment, the effects in high- and low-dose were highly concordant (24hr low-dose $\rho=.91, \mathrm{P}<2.2 \times 10^{-16} ; 6 \mathrm{hr}$ low-dose $\rho=.86, \mathrm{P}<2.2 \times 10^{-16}$ ) (S7C and S7D and S7E Fig).

We determined whether changes in gene expression in glucocorticoid signaling were driven through accessible chromatin, by testing for enrichment of glucocorticoid-responsive chromatin sites for proximity to differentially expressed genes. Glucocorticoid-responsive chromatin sites were significantly more likely to map within $100 \mathrm{~kb}$ of a gene with glucocorticoid-responsive expression compared to other chromatin sites in islets (high-dose: $\mathrm{OR}=1.48, \mathrm{P}=9.9 \times 10^{-}$ ${ }^{20}$; low-dose: $\left.\mathrm{OR}=4.91, \mathrm{P}=6.5 \times 10^{-36}\right)$. We next performed these analyses separately for sites up- and down-regulated in glucocorticoid signaling. There was significant enrichment of sites 
A

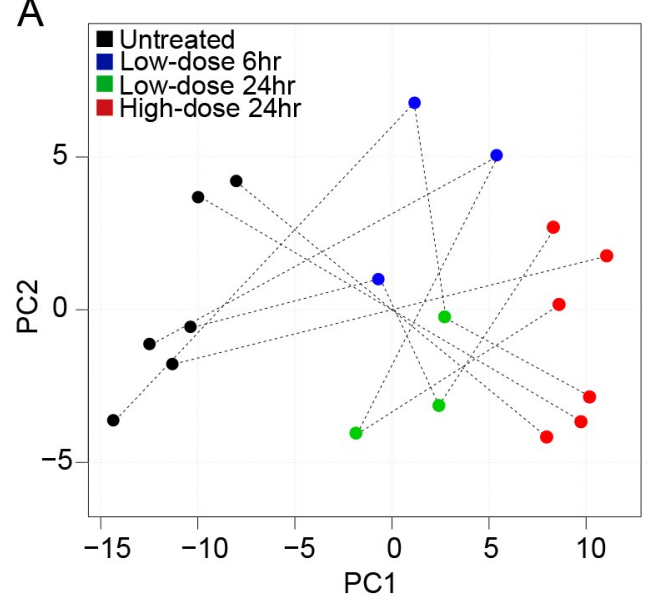

C

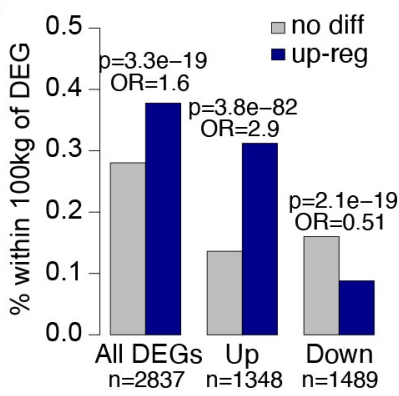

B

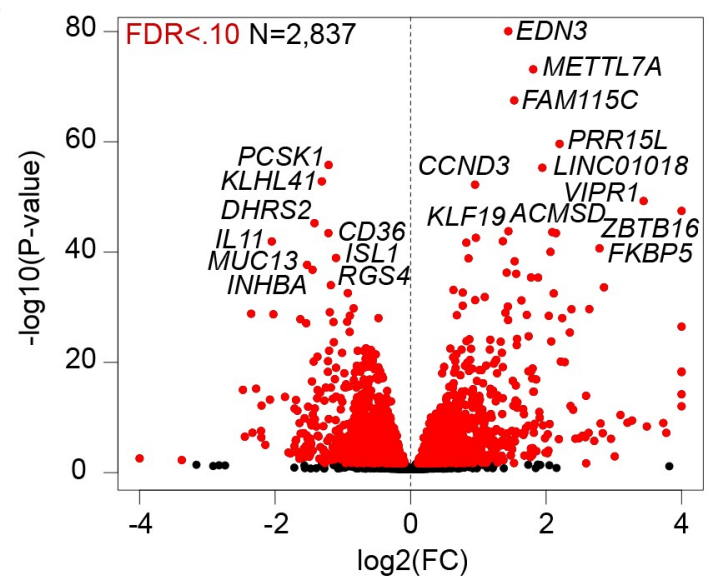

D
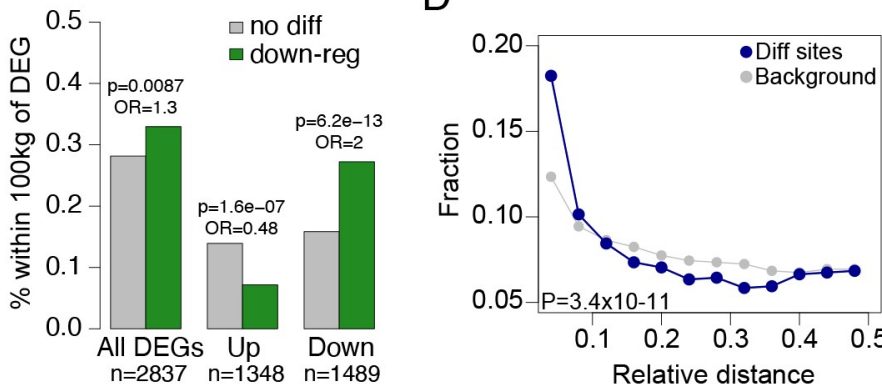

$E$

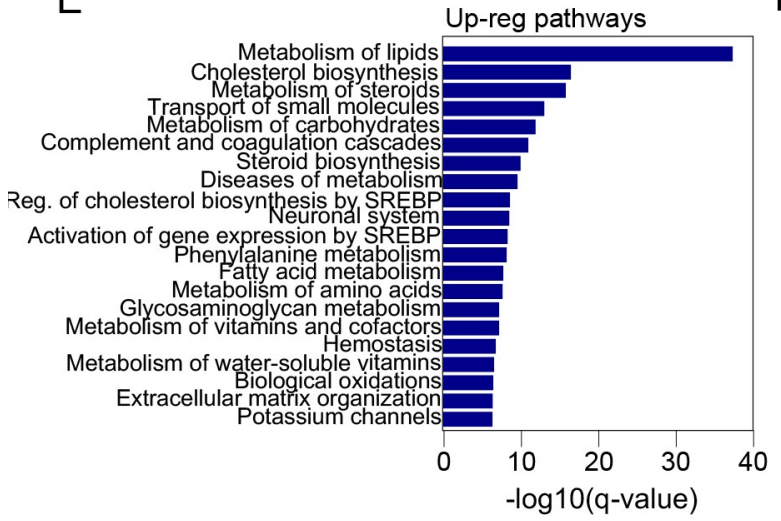

$\mathrm{F}$

Down-reg pathways

Cytokine signaling in immune system Signaling by receptor Pathways in cancer
Signaling by interleukins

Extracellular matrix organization

Nervous system development

Transport of small molecules

Cytokine-cytokine receptor interaction

Cellular responses to external stimuli

Signaling by nuclear receptors
Signaling by rho GTPases

Focal adhesion
Adaptive immune system

Segulation Signaling by VEGF

Resp. to elevated platelet cyto. Ca2

MAPK family signaling cascades

0

$10 \quad 20 \quad 30$ -log10(q-value)

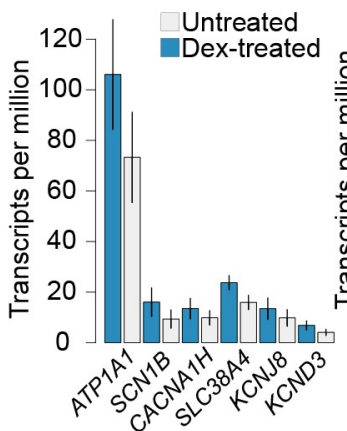

Ion transport

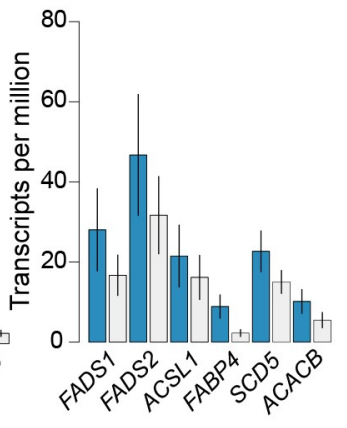

Lipid metabolism

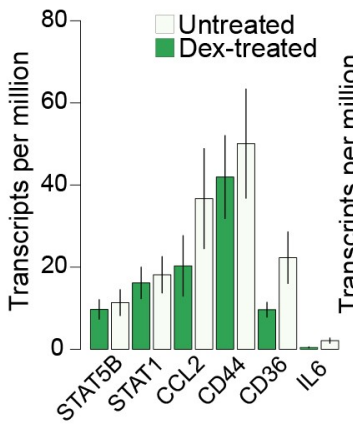

Inflammatory response

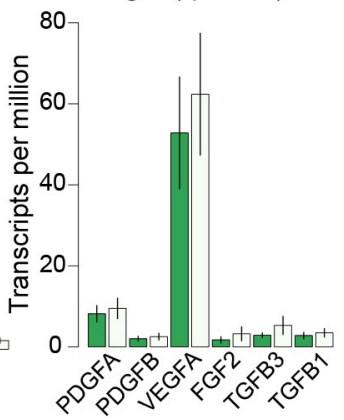

Proliferation

Fig 3. Glucocorticoid signaling affects gene expression levels in pancreatic islets. (A) Principal components plot of gene expression from high-dose (red) and low-dose (green $24 \mathrm{hr}$, blue $6 \mathrm{hr}$ ) glucocorticoid-treated and untreated (black) islets from a total of 6 samples. Dashed lines connect assays from the same sample. (B) Volcano plot showing genes with differential expression in 
glucocorticoid-treated islets compared to untreated islets. Genes with significantly differential expression $(\mathrm{FDR}<.10)$ are highlighted in red, and genes with pronounced changed in expression are listed. (C) Percentage of accessible chromatin sites with up-regulated activity (left) and down-regulated activity (right) in glucocorticoid-treated islets within 100kb of differentially expressed genes (DEGs) compared to chromatin sites without differential activity. (D) Relative distance metric (from bedtools reldist) between accessible chromatin sites with differential activity (dex) and genes with differential expression compared to all chromatin sites (background). (E) Biological pathway terms enriched among genes with up-regulated expression in glucocorticoidtreated islets (top), and the expression level of selected genes annotated with ion transport and lipid metabolism terms in glucocorticoid-treated and untreated islets (bottom). Values represent mean expression and standard error. (F) Biological pathway terms enriched among genes with up-regulated expression in glucocorticoid-treated islets (top), and the expression level of selected genes annotated with inflammatory response and proliferation pathway terms in glucocorticoid-treated and untreated islets (bottom). Values represent mean expression and standard error. For panels B, C, D and E the values shown are from results using high-dose treatment.

https://doi.org/10.1371/journal.pgen.1009531.g003

with increased activity in glucocorticoid signaling within $100 \mathrm{~kb}$ of genes with up-regulated expression specifically (up-reg $\mathrm{OR}=2.9, \mathrm{P}=3.8 \times 10^{-82}$, down-reg $\mathrm{OR}=0.51, \mathrm{P}=2.1 \times 10^{-19}$ ) (Fig 3C). Similarly, sites with decreased activity in glucocorticoid signaling were enriched within $100 \mathrm{~kb}$ of genes with down-regulated expression (down-reg $\mathrm{OR}=2.0, \mathrm{P}=6.2 \times 10^{-13}$, upreg $\mathrm{OR}=0.48, \mathrm{P}=1.6 \times 10^{-7}$ ) (Fig $3 \mathrm{C}$ ). Furthermore, we also observed an enrichment of glucocorticoid-responsive chromatin sites for closer proximity to genes with glucocorticoid-responsive expression compared to background sites (Kolmogorov-Smirnov $\mathrm{P}=3.4 \times 10^{-11}$ ) (Fig 3D).

In order to understand the molecular pathways affected by glucocorticoid activity in islets, we tested genes up- and down-regulated in glucocorticoid signaling for gene set enrichment using pathway and gene ontology (GO) terms (see Methods). Up-regulated genes in high-dose treatment were enriched for gene sets related to steroid metabolism (steroid metabolic process $\mathrm{FDR}=8.94 \times 10^{-30}$ ), lipid metabolism (lipid biosynthetic process FDR $=1.93 \times 10^{-32}$ ), potassium and other ion transport (potassium channels FDR $=5.71 \times 10^{-7}$; regulation of ion transport $\left.\mathrm{FDR}=1.93 \times 10^{-17}\right)$, and extracellular matrix organization $\left(\mathrm{FDR}=3.68 \times 10^{-7}\right)$ (Fig 3E and S7 Table). Similar gene sets were enriched among genes up-regulated in low-dose treatments (S7 Table). Numerous genes that function in ion transport were up-regulated in glucocorticoid signaling; for example ATP1A1, SCN1B, SCNN1A, CACNA1H, CACNG4, SLC38A4, TRPV6 as well as potassium channel genes including KCNJ2, KCNAB1, KCNF1, KCNJ8, and KCND3 (Fig 3E and $\mathrm{S} 6 \mathrm{Table}$ ). Up-regulated genes also included numerous that function in lipid metabolism including FADS1, FADS2, ACSL1, SCD5, FABP4, ACACB, and ANGPTL4 (Fig 3E and S6 Table).

Conversely, genes down-regulated in glucocorticoid signaling were enriched for inflammatory response (cytokine signaling in immune system $\mathrm{FDR}=2.2 \times 10^{-27}$, signaling by interleukins $\mathrm{FDR}=9.50 \times 10^{-19}$ ), extracellular matrix, cell adhesion and morphogenesis (extracellular matrix organization $\mathrm{FDR}=1.53 \times 10^{-17}$, regulation of cell adhesion $\mathrm{FDR}=2.48 \times 10^{-42}$, cellular component morphogenesis FDR $=2.45 \times 10^{-37}$ ), and cell differentiation and proliferation terms (neg. regulation of cell differentiation FDR $=2.18 \times 10^{-36}$ ) (Fig $3 \mathrm{~F}$ and S7 Table). Similar gene sets were enriched among genes down-regulated in low-dose treatments (S7 Table). Down-regulated genes included those involved in the inflammatory response such as IL6, STAT5B, STAT3, STAT4, SMAD3, CXCL12, CCL2, CD44, CD36, RELB, IRF1, extracellular matrix formation such matrix metalloproteinase genes such as $M M P 3, M M P 7, M M P 9$ and matrix components such as $L A M A 4$ and $L A M C 2$, islet function and pancreatic differentiation such as ISL1, PAX6, NKX6-1, HES1 and JAG1, and proliferation and growth factors such as PDGFA, $P D G F B, F G F 2$, TGFB3 and VEGFA (Fig 3F and S6 Table).

These results demonstrate that glucocorticoid signaling in islets up-regulates genes involved in steroid and lipid metabolism and ion channel activity, and down-regulates key genes in islet function as well as genes involved in inflammation, proliferation and extracellular matrix formation. 


\section{T2D and glucose associated variants map in glucocorticoid-responsive islet chromatin}

Genetic variants associated with diabetes risk are enriched in pancreatic islet regulatory elements. As these studies have been performed primarily using non-diabetic donors in normal (untreated) conditions, however, the role of environmental stimuli in modulating diabetes-relevant genetic effects on islet chromatin is largely unknown. We therefore tested for enrichment of diabetes and fasting glycemia associated variants in glucocorticoid-responsive islet chromatin sites using fgwas [40] (see Methods). We observed enrichment of variants influencing T2D risk and blood sugar (glucose) levels in chromatin sites with differential activity in both high- and low-dose glucocorticoid treatment (T2D high-dose $\ln ($ enrich) $=3.71,95 \%$ $\mathrm{CI}=3.03,4.25$; T2D low-dose $\ln ($ enrich $)=4.23,95 \% \mathrm{CI}=2.66,5.20$; blood sugar high-dose $\ln$ $($ enrich $)=3.92,95 \% \mathrm{CI}=0.86,5.70$; blood sugar low-dose $\ln ($ enrich $)=6.20,95 \% \mathrm{CI}=$ $3.92,8.42$ ) (Fig 4A). Conversely, we observed no evidence for enrichment of T1D risk variants (high-dose $\ln ($ enrich $)=-28.00,95 \% \mathrm{CI}=-48.00,3.39$; low-dose $\ln ($ enrich $)=-23.82,95 \% \mathrm{CI}=$ -43.8,5.29) (Fig 4A).

We next catalogued fine-mapped variants overlapping glucocorticoid-responsive islet chromatin using 99\% credible sets of T2D and glucose level signals from DIAMANTE and Biobank Japan (BBJ) [22,41] (see Methods). We identified 126 fine-mapped variants at 51 signals that overlapped a glucocorticoid-responsive site (S8 Table). We further identified 511 variants genome-wide in glucocorticoid-responsive sites with at least nominal evidence for T2D association $(\mathrm{P}<.005)$ in DIAMANTE or BBJ GWAS (S8 Table). We prioritized potential target genes of T2D- and glucose-associated variants in glucocorticoid-responsive chromatin by identifying genes proximal to these sites with differential expression. For example, T2D-associated variants at the 11q12 locus mapped in a site induced by glucocorticoids proximal to SCD5 and TMEM150C which both had up-regulated expression (Fig 4B and S3 and S8 Tables). Similarly, T2D-associated variants at the $4 \mathrm{q} 31$ locus mapped in a site down-regulated in glucocorticoids proximal to FBXW7 which had down-regulated expression (S7A Fig and S3 and S8 Tables). Outside of known T2D loci we observed additional examples such as at the 7p15 locus where rs1107376 $\left(\mathrm{T} 2 \mathrm{D} \mathrm{P}=2.2 \times 10^{-4}\right)$ mapped in a glucocorticoid-induced site proximal to $N P Y$ which had glucocorticoid-stimulated expression (S7B Fig and S3 and S8 Tables). At 71 T2D- or glucose-associated variants we further observed evidence for association with target gene expression (eQTL) in islets (S8 Table); for example, rs1107376 was an islet eQTL for NPY $\left(\mathrm{P}=2.2 \times 10^{-21}\right)$.

At the 2p21 locus associated with glucose level, lead variant rs12712928 (BBJ beta $=.068$, $\mathrm{P}=7.4 \times 10^{-46}$ ) mapped in a chromatin site with increased activity in glucocorticoid signaling and was proximal to SIX2 and SIX3 which both had glucocorticoid-induced expression (Fig $4 \mathrm{C}$ and $4 \mathrm{D}$ and $\mathrm{S} 8 \mathrm{Table}$ ). This variant had the highest posterior probability in fine-mapping data (PPA $=.89)$, suggesting it is likely causal for glucose association at this locus. This variant also had evidence for T2D association in BBJ (beta $=.048, \mathrm{P}=2.1 \times 10^{-6}$ ) and DIAMANTE (beta $=.022, \mathrm{P}=.012$ ), and was the lead variant at a T2D signal recently reported in East Asians $\left(\mathrm{P}=1.8 \times 10^{-14}\right)[42]$. We therefore tested whether $\mathrm{rs} 12712928$ affected enhancer activity using sequence around variant alleles in untreated and dexamethasone treated MIN6 cells (see Methods). The glucose increasing and T2D risk allele $\mathrm{C}$ had significantly reduced enhancer activity in both glucocorticoid-treated ( $\mathrm{T}$-test $\mathrm{P}=2.5 \times 10^{-6}$ ) and untreated cells ( $\mathrm{T}$-test $\mathrm{P}=3.2 \times 10^{-4}$ ) (Fig 4E). However, the allelic differences at this variant were more pronounced in glucocorticoid-treated cells (ref/alt ratio GC $=6.85,95 \% \mathrm{CI}=3.4,10.2$; untreated $=1.78,95 \% \mathrm{CI}=$ $1.23,2.32$, permutation test $\mathrm{P}=5.1 \times 10^{-3}$ ) (Fig $\left.4 \mathrm{~F}\right)$. We also observed evidence that $\mathrm{rs} 10168523$ was an islet eQTL for SIX3 and SIX2 (SIX3 P $=5.1 \times 10^{-23}$, SIX2 $\mathrm{P}=8.2 \times 10^{-10}$; Fig $\left.4 \mathrm{G}\right)$, where 


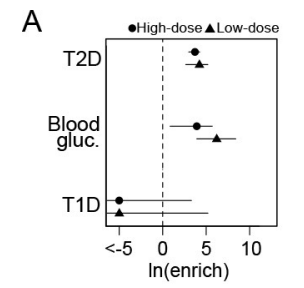

B

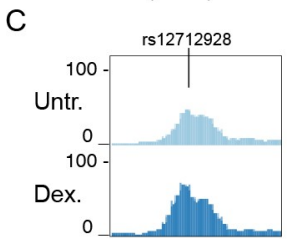

E

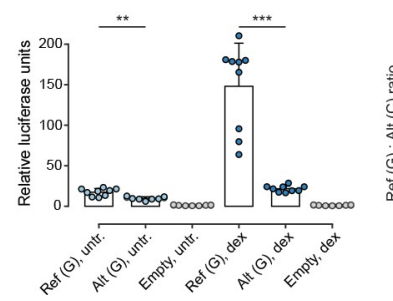

D chr4: $83,500,000|\quad 83,550,000| \quad 83,600,000|\quad 83,650,000| \quad 83,700,000 \mid$
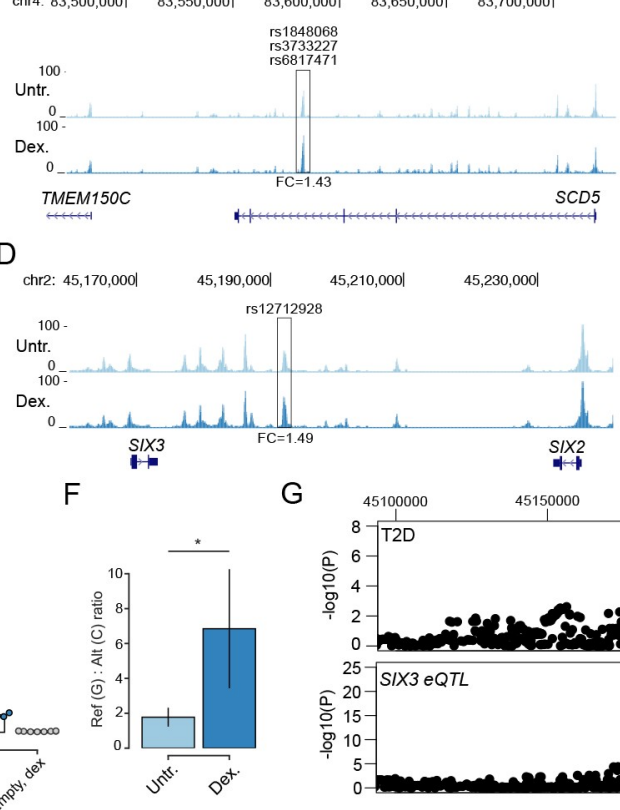
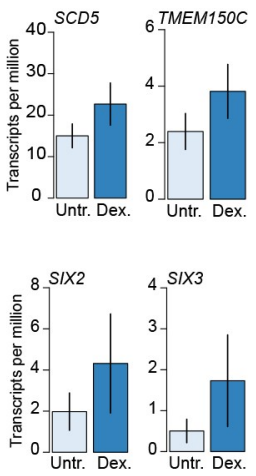

G

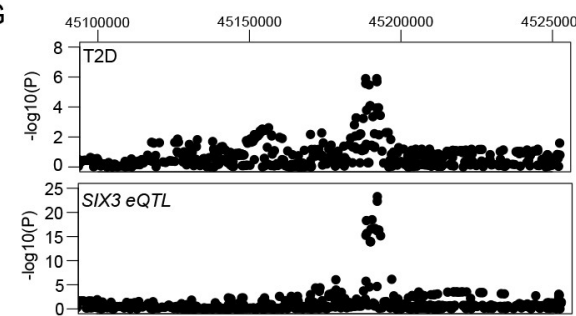

Fig 4. Type 2 diabetes and glucose associated variants affect glucocorticoid-responsive islet regulatory programs. (A) Enrichment of variants associated with type 1 diabetes (T1D), type 2 diabetes (T2D) and blood sugar (glucose) levels for differential chromatin sites in high-dose and low-dose glucocorticoid-treated islets. Values represent log enrichment estimates and 95\% confidence intervals. (B) Multiple fine-mapped T2D variants at the SCD5/TMEM150C locus mapped in a glucocorticoid-responsive islet accessible chromatin site. Both the SCD5 and TMEM150C genes had increased expression in glucocorticoid-treated islets. Genome browser tracks represent RPKM normalized ATAC-seq signal, and bar plots represent mean expression and standard error. (C, D) Variant rs12712928 with evidence for blood sugar and T2D association mapped in a glucocorticoid-responsive chromatin site at the SIX2/3 locus. Both the SIX2 and SIX3 genes had increased expression in glucocorticoid-treated islets. Genome browser tracks represent RPKM normalized ATAC-seq signal, and bar plots represent mean expression and standard error. (E) Variant rs12712928 had significant allelic effects on enhancer activity in gene reporter assays in MIN6 cells. Values represent mean and standard deviation. (F) The allelic effects of rs 12712928 were more pronounced in glucocorticoid-treated relative to untreated islets. Values represent fold-change and 95\% CI. (G) The T2D association signal at SIX2/3 was colocalized with an eQTL for SIX3 expression in islets. For panels B, C and D the values shown are from results using high-dose treatment. For panels B and D, the fold-change (FC) in accessible chromatin signal in glucocorticoid treatment compared to untreated is indicated at highlighted sites. ${ }^{* * *} \mathrm{P}<1 \times 10^{-4},{ }^{* *} \mathrm{P}<1 \times 10^{-3},{ }^{*} \mathrm{P}<1 \times 10^{-2}$.

https://doi.org/10.1371/journal.pgen.1009531.g004

the T2D risk allele was correlated with reduced expression of both genes. Glucose level and T2D association at this locus was strongly co-localized with the SIX3 and SIX2 eQTLs (BBJ T2D shared SIX3 PP = 89\%, SIX2 PP = 98\%; BBJ blood sugar shared SIX3 PP = 98\%, SIX2 $\mathrm{PP}=99 \%)($ Fig $4 \mathrm{G})$.

These results reveal that variants associated with T2D and glucose level are enriched in glucocorticoid-responsive chromatin sites in islets, including variants such as rs12712928 at the SIX2/3 locus which interact with glucocorticoid signaling directly to affect islet regulation.

\section{Discussion}

Our study demonstrates the relevance of islet chromatin dynamics in response to corticosteroid signaling to T2D pathogenesis, including T2D risk variants that interact with corticosteroid activity directly to affect islet chromatin. In a similar manner, variants mediating epigenomic responses of pancreatic islets to proinflammatory cytokines were recently shown to contribute to genetic risk of T1D [34]. Numerous environmental signals and external conditions modulate pancreatic islet function and contribute to the pathophysiology and genetic 
basis of diabetes, yet the epigenomic and transcriptional responses of islets to disease-relevant stimuli have not been extensively measured. Future studies of islet chromatin and gene regulation exposed to additional stimuli will therefore likely continue providing additional insight into diabetes risk.

Glucocorticoid signaling led to broad changes in accessible chromatin, which up-regulated the expression of proximal genes enriched for processes related to ion channels and transport, in particular potassium channels. Potassium ion concentrations modulate calcium influx and insulin secretion in beta cells [43], and in disruption of ion channel function leads to impaired glucose-induced insulin secretion and diabetes [44]. Glucocorticoids have been shown to suppress calcium influx while preserving insulin secretion via cAMP [7], and in line with this finding we observed evidence for increased activity of potassium channel and cAMP signaling genes and decreased activity of phosphodiesterase genes. Up-regulated genes were also strong enriched in lipid metabolism pathways, which has been shown to regulate insulin secretion and contribute to diabetes $[45,46]$. Several up-regulated genes PER1 and CRY2 are also components of the circadian clock, and previous studies have shown that endogenous glucocorticoid release is under control of circadian rhythms and therefore may contribute to downstream regulation of the clock [47]. Conversely, glucocorticoid signaling down-regulated inflammatory programs, in line with previous reports and the known function of glucocorticoids $[2,17,48]$, as well as key genes involved in islet function such as NKX6-1, PAX6, RFX6, and ISL1. Our findings further suggest that down-regulation of gene activity in glucocorticoid signaling is mediated through the activity of STAT and other TFs at proximal accessible chromatin sites, either through reduced TF expression or inhibition by GR. We also observed enrichment of FOXA binding in sites both up- and down-regulated in glucocorticoid signaling, suggesting these TFs mark sites that are broadly responsive to signal-dependent TF activity in islets in line with their known function as pioneer factors.

Genetic variants near the homeobox TFs SIX2 and SIX3 influence glucose levels [49,50], and our results provide evidence that both of these TFs operate downstream of glucocorticoid signaling and that the variants interact with this signaling program directly to influence glucose levels and risk of T2D. A previous study identified association between this locus and glucose levels in Chinese samples and demonstrated allelic effects of the same variant on islet enhancer activity and binding of the TF GABP [50], further supporting the likely causality of this variant. SIX2 and SIX3 have been widely studied for their role in forebrain, kidney and other tissue development [51-56]. In islets, both $S I X 2$ and $S I X 3$ have been shown to increase expression in adult compared to juvenile islets, and induction of SIX3 expression in EndoC- $\beta \mathrm{H} 1$ cells and juvenile islets enhanced islet function, insulin content and secretion and may contribute to the suppression of proliferative programs [57]. In line with this finding, the glucose-lowering and T2D protective allele of the likely causal variant increased islet enhancer activity and SIX2/3 expression.

Our in vitro experimental model mimics the environment of pancreatic islets under hormone signaling, albeit for a small number of treatments and conditions. Given the similarity in binding motifs of many nuclear hormone receptors and the enrichment of glucocorticoid responsive sites for androgen and progesterone receptor motifs, the effects of GR on islet gene regulation may overlap with other nuclear receptors by acting on shared chromatin sites [58]. Studies of other tissues have profiled glucocorticoid signaling across a broader range of experimental conditions and identified dose- and temporally-dependent effects on gene regulatory programs [14,15], and in islets dose- and temporally-dependent effects of glucocorticoids may impact insulin secretion and other islet functions. Future studies profiling the genomic activity of nuclear receptors in islets across a greater breadth of experimental conditions will therefore help further shed light into the role of hormone signaling dynamics in islet gene regulation and diabetes pathogenesis. 


\section{Methods}

\section{Ethics statement}

All studies were approved by the Institutional Review Board of the University of California San Diego.

\section{Human islet samples}

Human islet samples were obtained through the Integrated Islet Distribution Program (IIDP), University of Alberta and Prodo labs. Islet samples were further enriched using a dithizone stain. Islets were cultured for $24 \mathrm{hr}$ at approximately $10 \mathrm{~mL}$ media/ $1 \mathrm{k}$ islets in $10 \mathrm{~cm}$ dishes at 37C, 5\% CO2 in CMRL 1066 media supplemented with 10\% FBS, $1 \mathrm{X}$ pen-strep, 8mM glucose, $2 \mathrm{mM}$ L-glutamine, $1 \mathrm{mM}$ sodium pyruvate, $10 \mathrm{mM}$ HEPES, and $250 \mathrm{ng} / \mathrm{mL}$ Amphotericin $\mathrm{B}$. Treated islets had dexamethasone (Sigma) added in the culture media at either $100 \mathrm{ng} / \mathrm{mL}$ for $24 \mathrm{hr}, 4 \mathrm{ng} / \mathrm{mL}$ for $24 \mathrm{hr}$ or $4 \mathrm{ng} / \mathrm{mL}$ for $6 \mathrm{hr}$.

\section{ATAC-seq assays}

Islet samples were collected and centrifuged at 500xg for 3 minutes, then washed twice in HBSS, and resuspended in nuclei permeabilization buffer consisting of 5\% BSA, $0.2 \%$ IGEPAL-CA630, 1mM DTT, and 1X complete EDTA-free protease inhibitor (Sigma) in 1X PBS. Islets were homogenized using a chilled glass dounce homogenizer and incubated on a tube rotator for 10 mins before being filtered through a 30uM filter (sysmex) and centrifuged at $500 \mathrm{xg}$ in a $4 \mathrm{C}$ microcentrifuge to pellet nuclei. Nuclei were resuspended in Tagmentation Buffer (Illumina) and counted using a Countess II Automated Cell Counter (Thermo). Approximately 50,000 nuclei were transferred to a $0.2 \mathrm{~mL}$ PCR tube and volume was adjusted to $22.5 \mathrm{uL}$ with Tagmentation Buffer. 2.5uL TDE1 (Illumina) was added to each tagmentation reaction and mixed with gentle pipetting. Transposition reactions were incubated at $37 \mathrm{C}$ for 30 minutes. Tagmentation reactions were cleaned up using $2 \mathrm{X}$ reaction volume of Ampure XP beads (Beckman Coulter) and eluted in 20uL Buffer EB (Qiagen). 10uL tagmented DNA prepared as described above was used in a 25uL PCR reaction using NEBNext High-Fidelity Master Mix (New England Biolabs) and Nextera XT Dual-Indexed primers (Nextera). Final libraries were double size selected using Ampure XP beads and eluted in a final volume of 20uL Buffer EB. Libraries were analyzed using the Qubit HS DNA assay (Thermo) and Agilent 2200 Bioanalyzer (Agilent Biotechnologies). Sample libraries were sequenced on Illumina HiSeq 4000 using 100bp paired-end reads except for samples Isl10, Isl11 and Isl12 which were sequenced on Illumina NovaSeq 6000 using 100bp paired-end reads.

\section{RNA-seq assays}

RNA was isolated from treated and untreated islets using RNeasy Mini kit (Qiagen) and submitted to the UCSD Institute for Genomic Medicine to prepare and sequence ribodepleted RNA libraries. Sample libraries were sequenced on Illumina HiSeq4000 using 100bp pairedend reads except for samples Isl10, Isl11 and Isl12 which were sequenced on Illumina NovaSeq 6000 using 100bp paired-end reads.

\section{ATAC-seq data processing}

We trimmed reads using Trim Galore with options '-paired' and '-quality 10', then aligned them to the hg19 reference genome using BWA [59] mem with the '-M' flag. We then used samtools [60] to fix mate pairs, sort and index read alignments, used Picard (http:// broadinstitute.github.io/picard/) to mark duplicate reads, and used samtools [60] to filer reads 
with flags '-q 30', '-f 3', '-F 3332'. We then calculated the percentage of mitochondrial reads and percentage of reads mapping to blacklisted regions and removed all mitochondrial reads. We calculated a TSS enrichment score for each ATAC-seq experiment using the Python package 'tssenrich'. To obtain read depth signal tracks, we used bamCoverage [61] to obtain bigWig files for each alignment with signal normalization using RPKM.

\section{Identifying differential chromatin sites}

We first used Irreproducible Discovery Rate (IDR) to define a set of canonical ATAC-seq sites for differential analysis. In brief, for each condition separately, we pooled reads across all assays and randomly split the pooled reads into two 'pseudo-replicates'. For the pooled and 'pseudoreplicate' data we called candidate peaks using MACS2 [62] with the parameters '-extsize 150 -keep-dup all-shift -75 -nomodel -p 0.01'. We applied IDR to the 'pseudo-replicate' candidate peak calls and obtained the number of peaks at an IDR threshold of .01. We then sorted and filtered the pooled candidate peak calls based on this number. Finally, we merged the resulting peaks across conditions, where if two peaks overlapped, we retained the more significant peak, and considered these canonical sites for downstream analyses.

The set of alignments for each assay were then supplied as inputs to the $\mathrm{R}$ function featureCounts from the Rsubread [63] package to generate a matrix of read counts within each canonical site. We applied the R function DESeqDataSetFromMatrix from the DESeq2 [64] package to the read count matrix with default parameters then applied the DESeq function including donor as a variable to model paired samples. We considered sites differentially accessible with FDR $<0.1$, as computed by the Benjamini-Hochberg method.

We determined the percentage of differential sites with increased activity in glucocorticoids that overlapped a site active in untreated samples, as well as the percentage of differential sites proximal to a gene promoter defined as $5 \mathrm{~kb}$ upstream of the transcription start site.

\section{Principal components analysis}

We first defined input sites by merging overlapping (1bp or more) peaks identified in at least two experiments across all ATAC-seq experiments. We then constructed a read count matrix using edgeR [65] and calculated normalization factors using the 'calcNormFactors' function. We applied the voom transformation [66] and used the 'removeBatchEffect' function from limma [67] to regress out batch effects and sample quality effects (using TSS enrichment as a proxy for sample quality). We then restricted the read count matrix to the 100,000 most variable peaks and performed PCA analysis using the core R function 'prcomp' with rank 2.

\section{TF enrichment analysis}

Differentially accessible chromatin sites were analyzed for sequence motif enrichment compared to a background of all chromatin sites tested for differential activity using HOMER [68] and a masked hg19 reference genome with the command 'findMotifsGenome.pl $<$ bed file $>$ $<$ masked hg19>< > output dir $>$-bg $<$ background bed file $>$-size 200 -p 8 -bits -preparse -preparsedDir tmp'. We used the TF sequence motif database provided with the HOMER software. For TF ChIP-seq enrichment, we obtained ChIP-seq binding sites for 160 TFs generated by the ENCODE project [69] and tested for enrichment of binding in differential accessible chromatin sites compared to a background of all remaining chromatin sites genome-wide without differential activity. For each TF we calculated a $2 \times 2$ contingency table of overlap with differential sites and non-differential sites, determined significance using a Fisher test and calculated a fold-enrichment of overlap in differential compared to non-differential sites. 


\section{RNA-seq data processing and analysis}

Paired-end RNA-Seq reads were aligned to the genome using STAR [70] (2.5.3a) with a splice junction database built from the Gencode v19 gene annotation [71]. Gene expression values were quantified using the RSEM package (1.3.1) and filtered for $>0.1$ TPM on average per sample. Raw expression counts from the remaining 20,480 genes were normalized using variance stabilizing transformation (vst) from DESeq2 [64] and corrected for sample batch effects using limma removeBatchEffect. Principal component analysis was performed in $\mathrm{R}$ using the prcomp function. To identify differentially expressed genes between treated and untreated samples we obtained raw expression counts from RSEM [72] for the 20,480 genes and applied DESeq2 [64] with default settings including donor as a cofactor to model paired samples. To identify enriched GO terms in up and down-regulated genes, we applied GSEA [73] using Gene Ontology terms and KEGG/REACTOME pathway terms. We excluded gene sets with large numbers of genes in enrichment tests.

\section{Proximity of differential chromatin sites to differentially expressed genes}

We calculated the percentage of differential accessible chromatin sites mapping within 100kb of (i) all differentially expressed genes, (ii) up-regulated genes and (iii) down-regulated genes compared to non-differentially accessible sites, and determined the significance and odds ratio using a Fisher exact test. We calculated a relative distance metric with bedtools [74] (reldist function) using either differential chromatin sites or a background of all islet accessible chromatin sites as the "a" argument and differentially expressed genes as the "b" argument. We compared the distribution of relative distances from differential sites to the distribution from background sites using a Kolmogorov-Smirnov test.

\section{Sample genotyping and imputation}

Non-islet tissue was collected for seven samples during islet picking and used for genomic DNA extraction using the PureLink genomic DNA kit (Invitrogen). Genotyping was performed using Infinium Omni2.5-8 arrays (Illumina) at the UCSD Institute for Genomic Medicine. We called genotypes using GenomeStudio (v.2.0.4) with default settings. We then used PLINK [75] to filter out variants with 1) minor allele frequency (MAF) less than 0.01 in the Haplotype Reference Consortium (HRC) [76] panel r1.1 and 2) ambiguous A/T or G/C alleles with MAF greater than 0.4. For variants that passed these filters, we imputed genotypes into the HRC reference panel r1.1 using the Michigan Imputation Server with minimac4. Post imputation, we removed imputed genotypes with low imputation quality $(\mathrm{R} 2<.3)$.

\section{Allelic imbalance mapping}

We identified heterozygous variant calls in each sample with read depth of at least 10 in both untreated and treated cells, and then used WASP [77] to correct for reference mapping bias. We retained variants in each sample where both alleles were identified at least 3 times across untreated and treated cells. We then merged read counts at heterozygous SNPs from all samples in untreated and treated cells separately. We fit a beta-binomial model to the observed allele counts using the method of NPBin [78]. The parameters of the beta-binomial model were $\alpha=40.78$ and $\beta=39.26$ with over-dispersion of .012 for untreated samples and $\alpha=41.76$ and $\beta=40.10$ with over-dispersion of .012 for glucocorticoid-treated samples. We called imbalanced variants from the merged counts using a beta-binomial test, and then calculated qvalues from the resulting beta-binomial $\mathrm{p}$-values. We considered variants significant at FDR $<$ .10 . 


\section{Heterogeneous allelic imbalance}

For all variants with significant allelic imbalance in either glucocorticoid-treated or untreated conditions, we tested for heterogeneity in imbalance between conditions. We used Pearson's chi-squared test as implemented in the "prop.test" function of R. We calculated q-values from the resulting $\mathrm{p}$-values and considered variants significant at $\mathrm{FDR}<.10$.

\section{Genetic association analysis}

We tested glucocorticoid-responsive chromatin sites for enrichment of diabetes association using genome-wide association data for T1D [79], T2D from the DIAMANTE consortium [22], and blood sugar (glucose) from the Japan Biobank study [49]. For each study we retained variants with minor allele frequency (MAF) $>.05$ and tested for enrichment of high-dose and low-dose differential sites using fgwas [40] with a window size of $1 \mathrm{Mb}$.

We then cataloged all variants in glucocorticoid-responsive chromatin sites in T2D and glucose fine-mapping data and with nominal association $(\mathrm{P}<.005)$ genome-wide. For DIAMANTE, we used fine-mapping results provided with the study. For the Japan Biobank, we fine-mapped signals ourselves using summary statistics. We calculated approximate Bayes factors (ABF) for each variant as described previously [80]. We compiled index variants for each locus and defined variants within a $5 \mathrm{Mb}$ window and at least low linkage $\left(\mathrm{r}^{2}>0.1\right)$ in the East Asian subset of 1000 Genomes [81] with each index. For each variant, we calculated posterior probabilities of associations (PPA) by dividing the variant $\mathrm{ABF}$ by the sum of $\mathrm{ABF}$ for the locus. We defined $99 \%$ credible sets by sorting variants by descending PPA and retaining variants up to a cumulative probability of $99 \%$. For each variant in glucocorticoid-responsive chromatin, we identified protein-coding genes in GENCODE v33 with differential expression and where the gene body mapped within 100kb of the variant.

\section{Expression QTL analyses}

We obtained islet expression QTL data from a published study [82]. We extracted variant associations at the SIX2/SIX3 locus and tested for colocalization between T2D and blood sugar association in the Biobank Japan study and SIX2 and SIX 3 eQTLs using a Bayesian approach [83]. We considered signals colocalized with shared PP greater than $80 \%$.

\section{Gene reporter assays}

To test for allelic differences in enhancer activity at the SIX2/3 locus, we cloned human DNA sequences (Coriell) containing the reference allele upstream of the minimal promoter in the luciferase reporter vector pGL4.23 (Promega) using the enzymes Sac I and Kpn I. A construct containing the alternate allele was then created using the NEB Q5 SDM kit (New England Biolabs). The primer sequences used were as follows:

- Cloning FWD AGCTAGGTACCCCTCATCTGCCTTTCTGGAC

- Cloning REV TAACTGAGCTCCAGTGGGTATTGCTGCTTCC

- SDM FWD TGCATTGTTTcCTGTCCTGAAGACGAGC

- SDM REV GGGGGTGCCTGCATCTGC

MIN6 cells were seeded at approximately $2.5 \mathrm{E} 05$ cells $/ \mathrm{cm}^{\wedge} 2$ into a 48 -well plate. The day after passaging into the 48-well plate, cells were co-transfected with $250 \mathrm{ng}$ of experimental firefly luciferase vector pGL4.23 containing the alt or ref allele in the forward direction or an empty pGL4.23 vector, and 15ng pRL-SV40 Renilla luciferase vector (Promega) using the 
Lipofectamine 3000 reagent. Cells were fed culture media and stimulated where applicable 24 hours post-transfection. For stimulation $100 \mathrm{ng} / \mathrm{mL}$ dexamethasone (Sigma) was added to the culture media. Cells were lysed 48 hours post transfection and assayed using the Dual-Luciferase Reporter system (Promega). Firefly activity was normalized to Renilla activity and normalized results were expressed as fold change compared to the luciferase activity of the empty vector. The python package 'luciferase' was then used to remove batch effects. A two-sided ttest was used to compare the luciferase activity between the two alleles or between treatments. A permutation test was used to compare the allelic ratio of luciferase activity between the two treatments, based on 100,000 permutations of the allele labels.

\section{Supporting information}

S1 Fig. Gene expression in islets in response to different doses and durations of glucocorticoid treatment. Expression level of (A) ZBTB16, (B) VIPR1 and (C) IL11 in high-dose (100ng/ $\mathrm{mL}$ for $24 \mathrm{hr}$ ), low-dose ( $4 \mathrm{ng} / \mathrm{mL}$ for $24 \mathrm{hr}$ or $6 \mathrm{hr}$ ) glucocorticoid-treated or untreated islets. Values represent mean expression and standard error.

S2 Fig. Islet accessible chromatin signal across replicate samples at ZBTB16. RPKM normalized ATAC-seq signal for individual islet sample in high-dose glucocorticoid treated and untreated islets. Sites with differences in chromatin accessibility across conditions are highlighted.

S3 Fig. Islet accessible chromatin signal across replicate samples at VIPR1. RPKM normalized ATAC-seq signal for individual islet sample in high-dose glucocorticoid treated and untreated islets. Sites with differences in chromatin accessibility across conditions are highlighted.

S4 Fig. Accessible chromatin signal in islets in response to low dose glucocorticoid treatment. RPKM normalized ATAC-seq signal in low-dose ( $4 \mathrm{ng} / \mathrm{mL}$ for $6 \mathrm{hr}$ ) glucocorticoid treated and untreated islets at the (A) ZBTB16 and (B) VIPR1 loci. Sites induced by glucocorticoid treatment are highlighted.

S5 Fig. Islet accessible chromatin signal at IL11. RPKM normalized ATAC-seq signal in high-dose glucocorticoid treated and untreated islets at the IL11 locus. The IL11 promoter which has reduced accessibility in glucocorticoid treated islets at high dose is highlighted. (TIF)

S6 Fig. Differential chromatin accessibility in high- and low-dose glucocorticoid treatment. (A) Venn diagram of overlap in sites with differential activity in high-dose $(100 \mathrm{ng} / \mathrm{mL}$ for $24 \mathrm{hr}, \mathrm{n}=6)$ and low-dose $(4 \mathrm{ng} / \mathrm{mL}$ for $6 \mathrm{hr}, \mathrm{n}=3$ ) glucocorticoid treatment. (B) Effects of high-dose and low-dose glucocorticoid treatment on sites with significant differential activity in either treatment.

S7 Fig. Differential gene expression in high- and low-dose glucocorticoid treatment. (A,B) Volcano plot of differential gene expression in glucocorticoid-treated islets at low dose for $24 \mathrm{hr}$ or $6 \mathrm{hr}$ compared to untreated islets. Genes with significant differential expression (FDR $<.10$ ) are highlighted in red, and genes with most pronounced changes in expression are 
listed. (C) Venn diagram of overlap between genes differentially expressed in $24 \mathrm{hr}$ high $(\mathrm{n}=6), 24 \mathrm{hr}$ low $(\mathrm{n}=3), 6 \mathrm{hr}$ low $(\mathrm{n}=3)$ glucocorticoid treatment. (D) Effects of $24 \mathrm{hr}$ highand low-dose treatment on genes with significant differential expression in either treatment. (E) Effects of $24 \mathrm{hr}$ high- and $6 \mathrm{hr}$ low-dose treatment on genes with significant differential expression in either treatment.

S8 Fig. T2D-associated variants in differential chromatin sites. (A) Multiple variants at the FBXW7/TMEM154 locus mapped in a site with decreased activity and FBXW7 had decreased expression in glucocorticoid stimulation. (B) A variant at the NPY locus mapped in a site with increased activity and NPY had increased expression in glucocorticoid stimulation. Genome browser tracks represent RPKM normalized ATAC-seq signal, and expression bar plots represent mean expression and standard error. Values shown are from high-dose treatment. The fold-change (FC) in accessible chromatin signal in glucocorticoid treatment compared to untreated is indicated at highlighted sites.

S1 Table. Human islet donor samples. Islet samples used for genomic assays in this study and donor characteristics.

(XLSX)

S2 Table. Islet accessible chromatin sites. Complete list of 127,228 reproducible islet accessible chromatin sites identified by IDR. (XLSX)

S3 Table. Islet chromatin sites with differential activity in glucocorticoid treatment. List of islet accessible chromatin sites with differential activity in each treatment dose and duration using DESeq2.

(XLSX)

S4 Table. TFs enriched in differential chromatin sites. Sequence motifs and TF binding sites enriched in differential islet accessible chromatin sites in each treatment dose and duration. (XLSX)

S5 Table. Genetic variants with allelic imbalance in islet chromatin. List of variants with significant effects on accessible chromatin in untreated or glucocorticoid treated islets.

(XLSX)

S6 Table. Genes with differential expression in glucocorticoid-treated islets. Genes with differential expression in each treatment dose and duration using DESeq2. (XLSX)

S7 Table. Gene sets enriched in glucocorticoid-treated islets. Gene ontology and pathway terms enriched among genes with differential expression in each treatment dose and duration using GSEA.

(XLSX)

S8 Table. Diabetes risk variants in islet glucocorticoid-responsive chromatin sites. Genetic variants in $99 \%$ credible sets from fine-mapping data or with nominal association in genomewide summary statistic data from the DIAMANTE and Japan Biobank studies that mapped in islet accessible chromatin sites with differential activity.

(XLSX) 
S1 Data. Source data. Data underlying Figs 2F and 4E in the manuscript. (XLSX)

\section{Acknowledgments}

We thank the UC San Diego IGM Genomics Center for sequencing and technical support.

\section{Author Contributions}

Conceptualization: Kyle J. Gaulton.

Data curation: Anthony Aylward, Mei-Lin Okino, Paola Benaglio, Joshua Chiou, Elisha Beebe, Jose Andres Padilla, Sharlene Diep.

Formal analysis: Anthony Aylward, Mei-Lin Okino, Paola Benaglio, Joshua Chiou, Elisha Beebe, Kyle J. Gaulton.

Funding acquisition: Kyle J. Gaulton.

Investigation: Mei-Lin Okino, Jose Andres Padilla, Sharlene Diep.

Methodology: Anthony Aylward, Paola Benaglio, Joshua Chiou, Elisha Beebe.

Resources: Mei-Lin Okino.

Software: Anthony Aylward.

Supervision: Kyle J. Gaulton.

Writing - original draft: Anthony Aylward, Mei-Lin Okino, Paola Benaglio, Kyle J. Gaulton.

Writing - review \& editing: Anthony Aylward, Mei-Lin Okino, Paola Benaglio, Joshua

Chiou, Kyle J. Gaulton.

\section{References}

1. Becker DE. Basic and clinical pharmacology of glucocorticosteroids. Anesth Prog. 2013; 60: 25-31; quiz 32. https://doi.org/10.2344/0003-3006-60.1.25 PMID: 23506281

2. Coutinho $A E$, Chapman KE. The anti-inflammatory and immunosuppressive effects of glucocorticoids, recent developments and mechanistic insights. Mol Cell Endocrinol. 2011; 335: 2-13. https://doi.org/10. 1016/j.mce.2010.04.005 PMID: 20398732

3. Patel R, Williams-Dautovich J, Cummins CL. Minireview: new molecular mediators of glucocorticoid receptor activity in metabolic tissues. Mol Endocrinol Baltim Md. 2014; 28: 999-1011. https://doi.org/10. 1210/me.2014-1062 PMID: 24766141

4. Bauerle KT, Harris C. Glucocorticoids and Diabetes. Mo Med. 2016; 113: 378-383. PMID: 30228504

5. Suh S, Park MK. Glucocorticoid-Induced Diabetes Mellitus: An Important but Overlooked Problem. Endocrinol Metab Seoul Korea. 2017; 32: 180-189. https://doi.org/10.3803/EnM.2017.32.2.180 PMID: 28555464

6. Colvin ES, Ma H-Y, Chen Y-C, Hernandez AM, Fueger PT. Glucocorticoid-induced suppression of $\beta$ cell proliferation is mediated by Mig6. Endocrinology. 2013; 154: 1039-1046. https://doi.org/10.1210/ en.2012-1923 PMID: 23384834

7. Fine NHF, Doig CL, Elhassan YS, Vierra NC, Marchetti P, Bugliani M, et al. Glucocorticoids Reprogram $\beta$-Cell Signaling to Preserve Insulin Secretion. Diabetes. 2018; 67: 278-290. https://doi.org/10.2337/ db16-1356 PMID: 29203512

8. Gesina E, Tronche F, Herrera P, Duchene B, Tales W, Czernichow P, et al. Dissecting the role of glucocorticoids on pancreas development. Diabetes. 2004; 53: 2322-2329. https://doi.org/10.2337/diabetes. 53.9.2322 PMID: 15331541

9. Liu X, Turban S, Carter RN, Ahmad S, Ramage L, Webster SP, et al. $\beta$-Cell-Specific Glucocorticoid Reactivation Attenuates Inflammatory $\beta$-Cell Destruction. Front Endocrinol. 2014; 5: 165. https://doi org/10.3389/fendo.2014.00165 PMID: 25352830 
10. Lambillotte $C$, Gilon $P$, Henquin JC. Direct glucocorticoid inhibition of insulin secretion. An in vitro study of dexamethasone effects in mouse islets. J Clin Invest. 1997; 99: 414-423. https://doi.org/10.1172/ JCl119175 PMID: 9022074

11. Ullrich S, Berchtold S, Ranta F, Seebohm G, Henke G, Lupescu A, et al. Serum- and glucocorticoidinducible kinase 1 (SGK1) mediates glucocorticoid-induced inhibition of insulin secretion. Diabetes. 2005; 54: 1090-1099. https://doi.org/10.2337/diabetes.54.4.1090 PMID: 15793248

12. Oakley $\mathrm{RH}$, Cidlowski JA. The biology of the glucocorticoid receptor: new signaling mechanisms in health and disease. J Allergy Clin Immunol. 2013; 132: 1033-1044. https://doi.org/10.1016/j.jaci.2013. 09.007 PMID: 24084075

13. Reddy TE, Pauli F, Sprouse RO, Neff NF, Newberry KM, Garabedian MJ, et al. Genomic determination of the glucocorticoid response reveals unexpected mechanisms of gene regulation. Genome Res. 2009; 19: 2163-2171. https://doi.org/10.1101/gr.097022.109 PMID: 19801529

14. McDowell IC, Barrera A, D'Ippolito AM, Vockley CM, Hong LK, Leichter SM, et al. Glucocorticoid receptor recruits to enhancers and drives activation by motif-directed binding. Genome Res. 2018; 28: 12721284. https://doi.org/10.1101/gr.233346.117 PMID: 30097539

15. Vockley CM, D'Ippolito AM, McDowell IC, Majoros WH, Safi A, Song L, et al. Direct GR Binding Sites Potentiate Clusters of TF Binding across the Human Genome. Cell. 2016; 166: 1269-1281.e19. https:// doi.org/10.1016/j.cell.2016.07.049 PMID: 27565349

16. Ling J, Kumar R. Crosstalk between NFkB and glucocorticoid signaling: a potential target of breast cancer therapy. Cancer Lett. 2012; 322: 119-126. https://doi.org/10.1016/j.canlet.2012.02.033 PMID: 22433713

17. De Bosscher K, Vanden Berghe W, Vermeulen L, Plaisance S, Boone E, Haegeman G. Glucocorticoids repress NF-kappaB-driven genes by disturbing the interaction of p65 with the basal transcription machinery, irrespective of coactivator levels in the cell. Proc Natl Acad Sci U S A. 2000; 97: 3919-3924. https://doi.org/10.1073/pnas.97.8.3919 PMID: 10760263

18. Hazlehurst JM, Gathercole LL, Nasiri M, Armstrong MJ, Borrows S, Yu J, et al. Glucocorticoids fail to cause insulin resistance in human subcutaneous adipose tissue in vivo. J Clin Endocrinol Metab. 2013; 98: 1631-1640. https://doi.org/10.1210/jc.2012-3523 PMID: 23426618

19. Jubb AW, Boyle S, Hume DA, Bickmore WA. Glucocorticoid Receptor Binding Induces Rapid and Prolonged Large-Scale Chromatin Decompaction at Multiple Target Loci. Cell Rep. 2017; 21: 3022-3031. https://doi.org/10.1016/j.celrep.2017.11.053 PMID: 29241532

20. John S, Sabo PJ, Thurman RE, Sung M-H, Biddie SC, Johnson TA, et al. Chromatin accessibility predetermines glucocorticoid receptor binding patterns. Nat Genet. 2011; 43: 264-268. https://doi.org/10. 1038/ng.759 PMID: 21258342

21. Grøntved L, John S, Baek S, Liu Y, Buckley JR, Vinson C, et al. C/EBP maintains chromatin accessibility in liver and facilitates glucocorticoid receptor recruitment to steroid response elements. EMBO J. 2013; 32: 1568-1583. https://doi.org/10.1038/emboj.2013.106 PMID: 23665916

22. Mahajan A, Taliun D, Thurner M, Robertson NR, Torres JM, Rayner NW, et al. Fine-mapping type 2 diabetes loci to single-variant resolution using high-density imputation and islet-specific epigenome maps. Nat Genet. 2018; 50: 1505-1513. https://doi.org/10.1038/s41588-018-0241-6 PMID: 30297969

23. Fuchsberger C, Flannick J, Teslovich TM, Mahajan A, Agarwala V, Gaulton KJ, et al. The genetic architecture of type 2 diabetes. Nature. 2016; 536: 41-47. https://doi.org/10.1038/nature18642 PMID: 27398621

24. Gaulton KJ, Ferreira T, Lee Y, Raimondo A, Mägi R, Reschen ME, et al. Genetic fine mapping and genomic annotation defines causal mechanisms at type 2 diabetes susceptibility loci. Nat Genet. 2015; 47: 1415-1425. https://doi.org/10.1038/ng.3437 PMID: 26551672

25. Gaulton KJ. Mechanisms of Type 2 Diabetes Risk Loci. Curr Diab Rep. 2017; 17: 72. https://doi.org/10. 1007/s11892-017-0908-x PMID: 28741265

26. Pasquali L, Gaulton KJ, Rodríguez-Seguí SA, Mularoni L, Miguel-Escalada I, Akerman İ, et al. Pancreatic islet enhancer clusters enriched in type 2 diabetes risk-associated variants. Nat Genet. 2014; 46 : 136-143. https://doi.org/10.1038/ng.2870 PMID: 24413736

27. Chiou J, Zeng C, Cheng Z, Han JY, Schlichting M, Huang S, et al. Single cell chromatin accessibility reveals pancreatic islet cell type- and state-specific regulatory programs of diabetes risk. Genomics; 2019 Jul. https://doi.org/10.1101/693671

28. Gaulton KJ, Nammo T, Pasquali L, Simon JM, Giresi PG, Fogarty MP, et al. A map of open chromatin in human pancreatic islets. Nat Genet. 2010; 42: 255-259. https://doi.org/10.1038/ng.530 PMID: 20118932 
29. Stitzel ML, Sethupathy P, Pearson DS, Chines PS, Song L, Erdos MR, et al. Global epigenomic analysis of primary human pancreatic islets provides insights into type 2 diabetes susceptibility loci. Cell Metab. 2010; 12: 443-455. https://doi.org/10.1016/j.cmet.2010.09.012 PMID: 21035756

30. Varshney A, Scott LJ, Welch RP, Erdos MR, Chines PS, Narisu N, et al. Genetic regulatory signatures underlying islet gene expression and type 2 diabetes. Proc Natl Acad Sci U S A. 2017; 114: 2301-2306. https://doi.org/10.1073/pnas.1621192114 PMID: 28193859

31. Parker SCJ, Stitzel ML, Taylor DL, Orozco JM, Erdos MR, Akiyama JA, et al. Chromatin stretch enhancer states drive cell-specific gene regulation and harbor human disease risk variants. Proc Natl Acad Sci U S A. 2013; 110: 17921-17926. https://doi.org/10.1073/pnas.1317023110 PMID: 24127591

32. Greenwald WW, Chiou J, Yan J, Qiu Y, Dai N, Wang A, et al. Pancreatic islet chromatin accessibility and conformation reveals distal enhancer networks of type 2 diabetes risk. Nat Commun. 2019; 10: 2078. https://doi.org/10.1038/s41467-019-09975-4 PMID: 31064983

33. Miguel-Escalada I, Bonàs-Guarch S, Cebola I, Ponsa-Cobas J, Mendieta-Esteban J, Atla G, et al. Human pancreatic islet three-dimensional chromatin architecture provides insights into the genetics of type 2 diabetes. Nat Genet. 2019; 51: 1137-1148. https://doi.org/10.1038/s41588-019-0457-0 PMID: 31253982

34. Ramos-Rodríguez M, Raurell-Vila H, Colli ML, Alvelos MI, Subirana-Granés M, Juan-Mateu J, et al. The impact of proinflammatory cytokines on the $\beta$-cell regulatory landscape provides insights into the genetics of type 1 diabetes. Nat Genet. 2019; 51: 1588-1595. https://doi.org/10.1038/s41588-0190524-6 PMID: 31676868

35. Wasim M, Carlet M, Mansha M, Greil R, Ploner C, Trockenbacher A, et al. PLZF/ZBTB16, a glucocorticoid response gene in acute lymphoblastic leukemia, interferes with glucocorticoid-induced apoptosis. J Steroid Biochem Mol Biol. 2010; 120: 218-227. https://doi.org/10.1016/j.jsbmb.2010.04.019 PMID: 20435142

36. Wasim M, Mansha M, Kofler A, Awan AR, Babar ME, Kofler R. Promyelocytic leukemia zinc finger protein (PLZF) enhances glucocorticoid-induced apoptosis in leukemic cell line NALM6. Pak J Pharm Sci. 2012; 25: 617-621. PMID: 22713950

37. Fahnenstich J, Nandy A, Milde-Langosch K, Schneider-Merck T, Walther N, Gellersen B. Promyelocytic leukaemia zinc finger protein (PLZF) is a glucocorticoid- and progesterone-induced transcription factor in human endometrial stromal cells and myometrial smooth muscle cells. Mol Hum Reprod. 2003; 9 : 611-623. https://doi.org/10.1093/molehr/gag080 PMID: 12970399

38. Breen MS, Bierer LM, Daskalakis NP, Bader HN, Makotkine I, Chattopadhyay M, et al. Differential transcriptional response following glucocorticoid activation in cultured blood immune cells: a novel approach to PTSD biomarker development. TransI Psychiatry. 2019; 9: 201. https://doi.org/10.1038/s41398-0190539-x PMID: 31434874

39. Wang J, Zhu Z, Nolfo R, Elias JA. Dexamethasone regulation of lung epithelial cell and fibroblast interleukin-11 production. Am J Physiol. 1999; 276: L175-185. https://doi.org/10.1152/ajplung.1999.276.1. L175 PMID: 9887070

40. Pickrell JK. Joint analysis of functional genomic data and genome-wide association studies of 18 human traits. Am J Hum Genet. 2014; 94: 559-573. https://doi.org/10.1016/j.ajhg.2014.03.004 PMID: 24702953

41. Suzuki K, Akiyama M, Ishigaki K, Kanai M, Hosoe J, Shojima N, et al. Identification of 28 new susceptibility loci for type 2 diabetes in the Japanese population. Nat Genet. 2019; 51: 379-386. https://doi.org/ 10.1038/s41588-018-0332-4 PMID: 30718926

42. Spracklen CN, Horikoshi M, Kim YJ, Lin K, Bragg F, Moon S, et al. Identification of type 2 diabetes loci in 433,540 East Asian individuals. Genetics; 2019 Jun. https://doi.org/10.1101/685172

43. Rajan AS, Aguilar-Bryan L, Nelson DA, Yaney GC, Hsu WH, Kunze DL, et al. Ion channels and insulin secretion. Diabetes Care. 1990; 13: 340-363. https://doi.org/10.2337/diacare.13.3.340 PMID: 1689632

44. Jacobson DA, Shyng S-L. Ion Channels of the Islets in Type 2 Diabetes. J Mol Biol. 2019. https://doi. org/10.1016/j.jmb.2019.08.014 PMID: 31473158

45. Ye R, Gordillo R, Shao M, Onodera T, Chen Z, Chen S, et al. Intracellular lipid metabolism impairs $\beta$ cell compensation during diet-induced obesity. J Clin Invest. 2018; 128: 1178-1189. https://doi.org/10 1172/JCI97702 PMID: 29457786

46. Imai Y, Cousins RS, Liu S, Phelps BM, Promes JA. Connecting pancreatic islet lipid metabolism with insulin secretion and the development of type 2 diabetes. Ann N Y Acad Sci. 2020; 1461: 53-72. https:// doi.org/10.1111/nyas.14037 PMID: 30937918

47. Dickmeis T. Glucocorticoids and the circadian clock. J Endocrinol. 2009; 200: 3-22. https://doi.org/10. 1677/JOE-08-0415 PMID: 18971218 
48. King EM, Chivers JE, Rider CF, Minnich A, Giembycz MA, Newton R. Glucocorticoid repression of inflammatory gene expression shows differential responsiveness by transactivation- and transrepression-dependent mechanisms. PloS One. 2013; 8: e53936. https://doi.org/10.1371/journal.pone. 0053936 PMID: 23349769

49. Kanai M, Akiyama M, Takahashi A, Matoba N, Momozawa Y, Ikeda M, et al. Genetic analysis of quantitative traits in the Japanese population links cell types to complex human diseases. Nat Genet. 2018; 50: 390-400. https://doi.org/10.1038/s41588-018-0047-6 PMID: 29403010

50. Spracklen CN, Shi J, Vadlamudi S, Wu Y, Zou M, Raulerson CK, et al. Identification and functional analysis of glycemic trait loci in the China Health and Nutrition Survey. PLoS Genet. 2018; 14: e1007275. https://doi.org/10.1371/journal.pgen.1007275 PMID: 29621232

51. Carl M, Loosli F, Wittbrodt J. Six3 inactivation reveals its essential role for the formation and patterning of the vertebrate eye. Dev Camb Engl. 2002; 129: 4057-4063. PMID: 12163408

52. Brodbeck S, Besenbeck B, Englert C. The transcription factor Six2 activates expression of the Gdnf gene as well as its own promoter. Mech Dev. 2004; 121: 1211-1222. https://doi.org/10.1016/j.mod. 2004.05.019 PMID: 15327782

53. Lagutin OV, Zhu CC, Kobayashi D, Topczewski J, Shimamura K, Puelles L, et al. Six3 repression of Wnt signaling in the anterior neuroectoderm is essential for vertebrate forebrain development. Genes Dev. 2003; 17: 368-379. https://doi.org/10.1101/gad.1059403 PMID: 12569128

54. He G, Tavella S, Hanley KP, Self M, Oliver G, Grifone R, et al. Inactivation of Six2 in mouse identifies a novel genetic mechanism controlling development and growth of the cranial base. Dev Biol. 2010; 344: 720-730. https://doi.org/10.1016/j.ydbio.2010.05.509 PMID: 20515681

55. Samuel A, Rubinstein AM, Azar TT, Ben-Moshe Livne Z, Kim S-H, Inbal A. Six3 regulates optic nerve development via multiple mechanisms. Sci Rep. 2016; 6: 20267. https://doi.org/10.1038/srep20267 PMID: 26822689

56. Steinmetz PR, Urbach R, Posnien N, Eriksson J, Kostyuchenko RP, Brena C, et al. Six3 demarcates the anterior-most developing brain region in bilaterian animals. EvoDevo. 2010; 1: 14. https://doi.org/ 10.1186/2041-9139-1-14 PMID: 21190549

57. Arda HE, Li L, Tsai J, Torre EA, Rosli Y, Peiris H, et al. Age-Dependent Pancreatic Gene Regulation Reveals Mechanisms Governing Human $\beta$ Cell Function. Cell Metab. 2016; 23: 909-920. https://doi. org/10.1016/j.cmet.2016.04.002 PMID: 27133132

58. Reynolds MS, Hancock CR, Ray JD, Kener KB, Draney C, Garland K, et al. $\beta$-Cell deletion of Nr4a1 and $\mathrm{Nr} 4 \mathrm{a} 3$ nuclear receptors impedes mitochondrial respiration and insulin secretion. Am J Physiol Endocrinol Metab. 2016; 311: E186-201. https://doi.org/10.1152/ajpendo.00022.2016 PMID: 27221116

59. Li H, Durbin R. Fast and accurate short read alignment with Burrows-Wheeler transform. Bioinforma Oxf Engl. 2009; 25: 1754-1760. https://doi.org/10.1093/bioinformatics/btp324 PMID: 19451168

60. Li H, Handsaker B, Wysoker A, Fennell T, Ruan J, Homer N, et al. The Sequence Alignment/Map format and SAMtools. Bioinforma Oxf Engl. 2009; 25: 2078-2079. https://doi.org/10.1093/bioinformatics/ btp352 PMID: 19505943

61. Ramírez F, Ryan DP, Grüning B, Bhardwaj V, Kilpert F, Richter AS, et al. deepTools2: a next generation web server for deep-sequencing data analysis. Nucleic Acids Res. 2016; 44: W160-165. https://doi. org/10.1093/nar/gkw257 PMID: 27079975

62. Zhang Y, Liu T, Meyer CA, Eeckhoute J, Johnson DS, Bernstein BE, et al. Model-based analysis of ChIP-Seq (MACS). Genome Biol. 2008; 9: R137. https://doi.org/10.1186/gb-2008-9-9-r137 PMID: 18798982

63. Liao Y, Smyth GK, Shi W. The R package Rsubread is easier, faster, cheaper and better for alignment and quantification of RNA sequencing reads. Nucleic Acids Res. 2019; 47: e47. https://doi.org/10.1093/ nar/gkz114 PMID: 30783653

64. Love MI, Huber W, Anders S. Moderated estimation of fold change and dispersion for RNA-seq data with DESeq2. Genome Biol. 2014; 15: 550. https://doi.org/10.1186/s13059-014-0550-8 PMID: 25516281

65. Robinson MD, McCarthy DJ, Smyth GK. edgeR: a Bioconductor package for differential expression analysis of digital gene expression data. Bioinforma Oxf Engl. 2010; 26: 139-140. https://doi.org/10. 1093/bioinformatics/btp616 PMID: 19910308

66. Law CW, Chen Y, Shi W, Smyth GK. voom: Precision weights unlock linear model analysis tools for RNA-seq read counts. Genome Biol. 2014; 15: R29. https://doi.org/10.1186/gb-2014-15-2-r29 PMID: 24485249 
67. Ritchie ME, Phipson B, Wu D, Hu Y, Law CW, Shi W, et al. limma powers differential expression analyses for RNA-sequencing and microarray studies. Nucleic Acids Res. 2015; 43: e47. https://doi.org/10. 1093/nar/gkv007 PMID: 25605792

68. Heinz S, Benner C, Spann N, Bertolino E, Lin YC, Laslo P, et al. Simple combinations of lineage-determining transcription factors prime cis-regulatory elements required for macrophage and $\mathrm{B}$ cell identities. Mol Cell. 2010; 38: 576-589. https://doi.org/10.1016/j.molcel.2010.05.004 PMID: 20513432

69. ENCODE Project Consortium. An integrated encyclopedia of DNA elements in the human genome. Nature. 2012; 489: 57-74. https://doi.org/10.1038/nature11247 PMID: 22955616

70. Dobin A, Davis CA, Schlesinger F, Drenkow J, Zaleski C, Jha S, et al. STAR: ultrafast universal RNAseq aligner. Bioinforma Oxf Engl. 2013; 29: 15-21. https://doi.org/10.1093/bioinformatics/bts635 PMID: 23104886

71. Harrow J, Frankish A, Gonzalez JM, Tapanari E, Diekhans M, Kokocinski F, et al. GENCODE: the reference human genome annotation for The ENCODE Project. Genome Res. 2012; 22: 1760-1774. https://doi.org/10.1101/gr.135350.111 PMID: 22955987

72. Li B, Dewey CN. RSEM: accurate transcript quantification from RNA-Seq data with or without a reference genome. BMC Bioinformatics. 2011; 12: 323. https://doi.org/10.1186/1471-2105-12-323 PMID: 21816040

73. Subramanian A, Tamayo P, Mootha VK, Mukherjee S, Ebert BL, Gillette MA, et al. Gene set enrichment analysis: a knowledge-based approach for interpreting genome-wide expression profiles. Proc Natl Acad Sci U S A. 2005; 102: 15545-15550. https://doi.org/10.1073/pnas.0506580102 PMID: 16199517

74. Quinlan AR, Hall IM. BEDTools: a flexible suite of utilities for comparing genomic features. Bioinforma Oxf Engl. 2010; 26: 841-842. https://doi.org/10.1093/bioinformatics/btq033 PMID: 20110278

75. Purcell S, Neale B, Todd-Brown K, Thomas L, Ferreira MAR, Bender D, et al. PLINK: a tool set for whole-genome association and population-based linkage analyses. Am J Hum Genet. 2007; 81: 559 575. https://doi.org/10.1086/519795 PMID: 17701901

76. McCarthy S, Das S, Kretzschmar W, Delaneau O, Wood AR, Teumer A, et al. A reference panel of 64,976 haplotypes for genotype imputation. Nat Genet. 2016; 48: 1279-1283. https://doi.org/10.1038/ ng.3643 PMID: 27548312

77. van de Geijn B, McVicker G, Gilad Y, Pritchard JK. WASP: allele-specific software for robust molecular quantitative trait locus discovery. Nat Methods. 2015; 12: 1061-1063. https://doi.org/10.1038/nmeth. 3582 PMID: 26366987

78. Zhang Q, Keles S. An empirical Bayes test for allelic-imbalance detection in ChIP-seq. Biostat Oxf Engl. 2018; 19: 546-561. https://doi.org/10.1093/biostatistics/kxx060 PMID: 29126153

79. Aylward A, Chiou J, Okino M-L, Kadakia N, Gaulton KJ. Shared genetic risk contributes to type 1 and type 2 diabetes etiology. Hum Mol Genet. 2018. https://doi.org/10.1093/hmg/ddy314 PMID: 30407494

80. Wakefield J. A Bayesian measure of the probability of false discovery in genetic epidemiology studies. Am J Hum Genet. 2007; 81: 208-227. https://doi.org/10.1086/519024 PMID: 17668372

81. 1000 Genomes Project Consortium, Auton A, Brooks LD, Durbin RM, Garrison EP, Kang HM, et al. A global reference for human genetic variation. Nature. 2015; 526: 68-74. https://doi.org/10.1038/ nature15393 PMID: 26432245

82. Viñuela A, Varshney A, van de Bunt M, Prasad RB, Asplund O, Bennett A, et al. Genetic variant effects on gene expression in human pancreatic islets and their implications for T2D. Nat Commun. 2020; 11: 4912. https://doi.org/10.1038/s41467-020-18581-8 PMID: 32999275

83. Giambartolomei C, Vukcevic D, Schadt EE, Franke L, Hingorani AD, Wallace C, et al. Bayesian test for colocalisation between pairs of genetic association studies using summary statistics. PLoS Genet. 2014; 10: e1004383. https://doi.org/10.1371/journal.pgen.1004383 PMID: 24830394 